\title{
A Study of the Plant Folk Nomenclature of the Yi People in Xiaoliangshan, Yunnan Province, China, and the Implications for Protecting Biodiversity
}

\section{Yi-Won A·D \\ University of Chinese Academy of Sciences}

\section{Yu Zhang}

Yunnan Key Laboratory for Wild Plant Resources, Kunming Institute of Botany, Chinese Academy of Sciences, Kunming, China

\section{Xiao-Yong Ding}

University of Chinese Academy of Sciences

\section{Chang-An Guo}

University of Chinese Academy of Sciences

Yu-Hua Wang ( $\nabla$ wangyuhua@mail.kib.ac.cn )

Yunnan Key Laboratory for Wild Plant Resources, Kunming Institute of Botany, Chinese Academy of Sciences, Kunming, China

\section{Research Article}

Keywords: Ethnobotany, Yi people, Xiaoliangshan, Indigenous botanical nomenclature

Posted Date: November 10th, 2021

DOl: https://doi.org/10.21203/rs.3.rs-1052221/v1

License: () (1) This work is licensed under a Creative Commons Attribution 4.0 International License. Read Full License 


\section{Abstract}

\section{Background}

Folk plant nomenclature is a part of knowledge of indigenous people often used to distinguish plant species. This study aimed to document the folk botanical nomenclature of the Yi people in Xiaoliangshan, north-west Yunnan Province, which has not been systematically investigated to date. The results of this study will assist in conserving biodiversity and the language of the Yi people and will promote the transmission of ethnobotanical knowledge.

\section{Methods}

An ethnobotanical survey of plants used by the Yi people in Xiaoliangshan, Yunnan Province, was conducted from September 2019 to August 2021. Semi-structured Interviews and Key Informant Interviews were conducted to collect and document ethnobotanical information, which was then used to analyse the folk botanical nomenclature of the Yi people. In addition, the folk names of plants used by the Xiaoliangshan Yi community were compared with those of the Yi people living in the Daliangshan, where the environment is considerably different.

\section{Results}

In this study, 266 informants were interviewed, and the names of 228 indigenous plants were extracted from 3088 use reports. The nomenclature used by the local Yi people is based on plant characteristics, plant habitat, plant-use, and the local culture. The folk names and the plant species have three types of correspondence. Moreover, some plant names are related to animals or Chinese loanwords. The comparison between the folk botanical names used by the Yi people in Xiaoliangshan and those used by the Yi people in the Daliangshan shows that 55 names are identical and the Yi people in both locations use 41 identical plants.

\section{Conclusions}

There are certain similarities and differences between the folk nomenclature of the Yi people in Xiaoliangshan and those in the Daliangshan. The findings are an example of how language evolves when people from the same ethnic group live in different geographical environments for extensive periods. In addition, the numerous Chinese loanwords in the folk botanical names of the Yi people in both locations indicate that a loss of traditional knowledge may be one of the greatest challenges to preserving the cultural heritage of the Yi people and to the conservation of biodiversity.

\section{Introduction}

Plants have been studied and used throughout human history, and the vast number of botanical names in different languages attests to human plant knowledge [1]. Almost all cultures have names for indigenous plants [2], and as a unique naming system based on traditional ethnobotanical knowledge and indigenous language, folk botanical nomenclature reflects the linguistic rules and cultural phenomena of the local 
population. Therefore, folk botanical nomenclature is an important resource that enables locals to recognise, remember and use plants, and ultimately to protect plant diversity [3]. Understanding and elucidating folk nomenclature of local plant species is an important part of ethnobotanical and anthropological research [47]. Many studies in China have focused on the folk botanical nomenclature of the Dai $[8,9,2,10]$ and the Mongolians [11-13]. Some researchers have documented the plant nomenclature of the Yi people in the Daliangshan Yi Autonomous Prefecture in Sichuan Province [3], where the Yi people often use monosyllabic words to name culturally important plants but use Chinese loanwords to name introduced species. The plantnaming system of the Yi people uses binomial and non-binomial forms, and a recent study on plants used in the Bimo religious rituals of the Yi people in Xiaoliangshan [14] found that plants with both binomial and non-binomial names were employed in these rituals. However, this study focused only on the use of plants by the Yi people from the perspective of religious rituals, and it is unclear whether the same nomenclature is employed for plants used for other purposes.

Hengduan Mountains, which are a popular area for studying biodiversity. The combination of the monsoon climate and the complex mountain environment makes it one of the most abundant alpine flora regions in the world $[15,16]$. Various ethnic groups who live in this region, including the Yi people, depend on the region's flora for survival: plants are used for medicine, food, feed, fuel, dyes, spices, landscaping, religious ceremonies, and other purposes [17-19]. Xiaoliangshan lies in the north-western part of Yunnan Province within the Hengduan Mountains. The Yi people living in Xiaoliangshan progressively migrated there from the Daliangshan and they now constitute the main ethnic group in this area [20, 21].

Academic research on the Xiaoliangshan Yi people has focused primarily on the cultural heritage of the Yi ethnic group from the perspective of anthropology [20,21,22], whereas no systematic research has investigated their ethnobotanical knowledge. Combined ethnobotanic and anthropologic studies of the $\mathrm{Yi}$ ethnic group would enable the folk botanical nomenclature used by the Yi community in Xiaoliangshan to be established, and such research would contribute to preserving traditional botanical knowledge and promoting and protecting biodiversity within this region.

Therefore, this study aimed to document and analyse the folk botanical nomenclature of the Yi ethnic group in Xiaoliangshan. We aimed to answer the two following questions: (1) What are the rules for the plant nomenclature used by the Yi people in Xiaoliangshan? (2) What are the similarities and differences between the plant folk nomenclature of the Xiaoliangshan Yi people and those of the Yi people in the Daliangshan, who have the same cultural heritage, but live in a different environment? This paper examines the significance of their plant nomenclature methods and the effect that folk botanical nomenclature has on protecting biodiversity and preserving traditional ethnobotanical knowledge.

\section{Methods}

\subsection{Study area and introduction to the Yi people}

Xiaoliangshan (lat. $26^{\circ} 36^{\prime}-27^{\circ} 56^{\prime} \mathrm{N}$; long. $100^{\circ} 22^{\prime}-101^{\circ} 15^{\prime} \mathrm{E}$ ) is situated in the northwest of Yunnan Province within the middle section of the Hengduan Mountains (Figure 1). It lies on the border of Sichuan and Yunnan province and has a temperate monsoon climate characterised by warm and moist summers, cold and dry 
winters, and four distinct seasons [23]. Its primary soil types are subalpine meadow soil, dark brown soil, and subalpine desert soil [24]. Due to its unique geographical location and climatic conditions, there is abundant and diverse flora within the area.

In this study, we conducted ethnobotanical research in 14 villages and 3 communities within six townships in the eastern part of Xiaoliangshan (Table 1). The Yi people are the main ethnic group within the selected research location, and their traditional lifestyle is well preserved in these communities. According to some studies, the Yi people progressively migrated to Xiaoliangshan from the Daliangshan, and they have eventually become the main ethnic group in this region $[20,21]$. In the early $19^{\text {th }}$ century, the Yi people in Xiaoliangshan made a living through animal husbandry, farming, and hunting and gathering [25]. Traditional Yi dwellings are made of wood or clay-and-wood [26], and their staple foods include potato, buckwheat, oats, corn, and turnip [27]. Grilling and boiling are commonly used cooking methods [27]. The Yi people firmly believe in animism and worship nature. They also believe that all living things originate from snow, which they consider to be the common ancestor of animals and plants [28]. In the Bimo belief system, the Bimo (a ritual specialist or priest) presides over all major religious activities, including offering prayers and sacrifices $[29,30]$. The Yi people in Xiaoliangshan have their own language and script and they use the northern Yi dialect in their daily communication [31].

Table 1

Surveyed locations within study area. 


\begin{tabular}{|c|c|c|c|c|c|}
\hline Town & Village/community & Longitude & Latitude & Altitude $\rrbracket \mathrm{m} \square$ & Population \\
\hline Dàxing town & $\begin{array}{l}\text { Well-off homes } \\
\text { community }\end{array}$ & $100.861411 \mathrm{E}$ & $27.304879 \mathrm{~N}$ & 2255 & 2329 \\
\hline Dàxing town & $\begin{array}{l}\text { Riverside Homes } \\
\text { Community }\end{array}$ & 100.865977E & $27.284771 \mathrm{~N}$ & 2255 & 2840 \\
\hline Dàxing town & $\begin{array}{l}\text { Happy Homes } \\
\text { Community }\end{array}$ & 100.864976E & $27.306978 \mathrm{~N}$ & 2255 & 6613 \\
\hline Nínglì township & Nínglì Village & 100.765049E & $27.251272 \mathrm{~N}$ & 2400 & 4956 \\
\hline Nínglì township & Báicăopíng Village & $100.71238 \mathrm{E}$ & $27.174713 \mathrm{~N}$ & 2400 & 2043 \\
\hline $\begin{array}{l}\text { Lànníqìng } \\
\text { township }\end{array}$ & Lànníqìng Village & 100.983124E & $27.225657 \mathrm{~N}$ & 2850 & 2891 \\
\hline $\begin{array}{l}\text { Lànníqing } \\
\text { township }\end{array}$ & Dàerdì Village & 100.940823E & $27.275785 \mathrm{~N}$ & 2750 & 2398 \\
\hline $\begin{array}{l}\text { Xinyýngpán } \\
\text { township }\end{array}$ & Xīnyíngpán Village & $100.926102 E$ & $27.172216 \mathrm{~N}$ & 2500 & 4476 \\
\hline $\begin{array}{l}\text { Xinyíngpán } \\
\text { township }\end{array}$ & Dōng fēng Village & 100.919985E & $27.187754 \mathrm{~N}$ & 2654 & 3441 \\
\hline $\begin{array}{l}\text { Xīnyíngpán } \\
\text { township }\end{array}$ & Máojiāxiāng Village & $100.945282 E$ & $27.138304 \mathrm{~N}$ & 2600 & 4052 \\
\hline $\begin{array}{l}\text { Pǎomǎpíng } \\
\text { township }\end{array}$ & Păomăpíng Village & $100.987172 \mathrm{E}$ & $26.996425 \mathrm{~N}$ & 2680 & 4009 \\
\hline $\begin{array}{l}\text { Pǎomăpíng } \\
\text { township }\end{array}$ & Shālìpíng Village & 101.013091E & $26.969145 \mathrm{~N}$ & 2720 & 3297 \\
\hline $\begin{array}{l}\text { Pǎomăpíng } \\
\text { township }\end{array}$ & Yángchăng Village & 101.045571E & $26.937666 \mathrm{~N}$ & 2480 & 1728 \\
\hline $\begin{array}{l}\text { Chánzhànhé } \\
\text { township }\end{array}$ & Chánzhànhé Village & 101.180402E & $26.98326 \mathrm{~N}$ & 2900 & 4163 \\
\hline $\begin{array}{l}\text { Chánzhànhé } \\
\text { township }\end{array}$ & Sāngǔshǔi Village & 101.077553E & $26.973122 \mathrm{~N}$ & 2900 & 1627 \\
\hline $\begin{array}{l}\text { Chánzhànhé } \\
\text { township }\end{array}$ & Gànhăiži Village & 101.135092E & $27.066066 \mathrm{~N}$ & 1680 & 1387 \\
\hline $\begin{array}{l}\text { Chánzhànhé } \\
\text { township }\end{array}$ & Wànmăchăng Village & 101.095586E & $27.033905 \mathrm{~N}$ & 2900 & 923 \\
\hline
\end{tabular}

\subsection{Ethnobotanical survey and data collection}

We conducted several systematic ethnobotanical surveys and investigations in Xialoiangshan from September 2019 to August 2021. We used snowball sampling to recruit a total of 266 informants, including 151 males and 115 females. The informants held various occupations, such as local farmers and herdsmen, 
Bimo practitioners, students, forest rangers, and folk doctors. Key informant interviews and semi-structured interviews were conducted with the informants upon their consent. The interviews were conducted at the informants' homes, fields, shrub, and pine forests, and at sacrificial ritual locations. The first author of this article is a local member of the $Y i$ ethnic group, whose mother tongue is the $Y i$ language. To facilitate communication with the informants and ensure the integrity of the acquired information, all interviews were conducted and documented in Yi language. During each interview, the informants were asked the following pre-prepared questions: (1) What plants do you usually use and how do you use them? (2) What are their names? (3) Can you explain the meaning of their names?

Finally, voucher specimens of the different plants were collected in the nearby fields, farmland, and along roadsides, under the guidance of the key informants. All the collected voucher specimens were authenticated by each member of the research team in charge of this study, based on the publication "Flora of China" [32] and then stored at the Herbarium of the Kunming Institute of Botany, Chinese Academy of Sciences.

\subsection{Data analysis}

After informant interviews, Microsoft Excel 2016 (Microsoft Corporation, http://www.microsoft.com/) was used to compile the collected data. Acai Yi input (https://www.cr173.com/soft/642454.html『was employed to transcribe the handwritten notes into the corresponding Excel tables. The information collected in the informant interviews served as the basis for our research on the folk botanical nomenclature and classification rules of the Yi people in Xiaoliangshan.

\section{Results}

\subsection{Plant species used by the Yi community in Xiaoliangshan}

We collected a total of 3088 use reports and extracted 228 folk names of local plants, belonging to 107 families, 178 genera, and 226 species (Table 2). The record of each useful plant includes the following information: plant name in the Yi language and Yi language phonetic name, Latin name, family name of the plant species, voucher specimen number, and the number of use reports.

Table 2

Catalogue of plants used by the Yi people in Xiaoliangshan, Yunnan Province. 


\begin{tabular}{|c|c|c|c|c|c|}
\hline $\begin{array}{l}\text { Yi } \\
\text { language } \\
\text { name }\end{array}$ & $\begin{array}{l}\text { Yi } \\
\text { language } \\
\text { phonetic } \\
\text { name }\end{array}$ & Latin name & Family & $\begin{array}{l}\text { The } \\
\text { number } \\
\text { of use } \\
\text { reports }\end{array}$ & $\begin{array}{l}\text { Voucher } \\
\text { number }\end{array}$ \\
\hline 吅 & map bu & Paris polyphylla Smith & Melanthiaceae & 215 & $\begin{array}{l}\text { QTP- } \\
\text { EBT5000 }\end{array}$ \\
\hline 吅 & hxi ke & Artemisia argyi Lévl. et Van. & Asteraceae & 165 & $\begin{array}{l}\text { QTP- } \\
\text { EBT5001 }\end{array}$ \\
\hline 吅 & yie pie & Papaver somniferum L. & Papaveraceae & 113 & \\
\hline 吅 & $\begin{array}{l}\text { va ddot } \\
\text { chu }\end{array}$ & Berberis pruinosa Franch. & Berberidaceae & 106 & $\begin{array}{l}\text { QTP- } \\
\text { EBT5002 }\end{array}$ \\
\hline 吅 & chup nuop & Prinsepia utilis Royle & Rosaceae & 90 & $\begin{array}{l}\text { QTP- } \\
\text { EBT5003 }\end{array}$ \\
\hline प्राप्रा & $\begin{array}{l}\text { a jji bap } \\
\text { mop }\end{array}$ & $\begin{array}{l}\text { Dipsacus asper Wallich ex } \\
\text { Candolle }\end{array}$ & Caprifoliaceae & 87 & $\begin{array}{l}\text { QTP- } \\
\text { EBT5004 }\end{array}$ \\
\hline प्राप्रा & $\begin{array}{l}\text { va bu syt } \\
\text { pup ssut }\end{array}$ & Taxillus Tiegh.sp & Loranthaceae & 71 & $\begin{array}{l}\text { QTP- } \\
\text { EBT5005 }\end{array}$ \\
\hline प्राप्रा & $\begin{array}{l}\text { vot mop } \\
\text { ddie bbur }\end{array}$ & Plantago major L. & Plantaginaceae & 70 & $\begin{array}{l}\text { QTP- } \\
\text { EBT5006 }\end{array}$ \\
\hline 吅 & bbit yop & Bulbophyllum Thouars.sp & Orchidaceae & 69 & $\begin{array}{l}\text { QTP- } \\
\text { EBT5007 }\end{array}$ \\
\hline 吅 & chup tu & $\begin{array}{l}\text { Rubus biflorus Buch. -Ham. ex } \\
\text { Smith }\end{array}$ & Rosaceae & 68 & $\begin{array}{l}\text { QTP- } \\
\text { EBT5008 }\end{array}$ \\
\hline प्राप्रा & $\begin{array}{l}\text { va zza } \\
\text { hnap zzy }\end{array}$ & Potentilla lineata Treviranus & Rosaceae & 63 & $\begin{array}{l}\text { QTP- } \\
\text { EBT5009 }\end{array}$ \\
\hline 吅 & $\begin{array}{l}\text { chup nuop } \\
\text { ssut }\end{array}$ & Taxillus.sp & Loranthaceae & 59 & $\begin{array}{l}\text { QTP- } \\
\text { EBT5010 }\end{array}$ \\
\hline 吅 & sy qi ddi & Sambucus williamsii Hance & Adoxaceae & 58 & $\begin{array}{l}\text { QTP- } \\
\text { EBT5011 }\end{array}$ \\
\hline वाप्रा & jy sy ssut & $\begin{array}{l}\text { Taxillus delavayi (Van Tiegh.) } \\
\text { Danser }\end{array}$ & Loranthaceae & 53 & $\begin{array}{l}\text { QTP- } \\
\text { EBT5012 }\end{array}$ \\
\hline प्राप्रा & $\begin{array}{l}\text { vot mop } \\
\text { zza ke }\end{array}$ & Crepis lignea (Vaniot) Babcock & Asteraceae & 49 & $\begin{array}{l}\text { QTP- } \\
\text { EBT5013 }\end{array}$ \\
\hline वस्पा & $\begin{array}{l}\text { syp ap } \\
\text { mop }\end{array}$ & Lonicera calcarata Hemsl. & Caprifoliaceae & 48 & $\begin{array}{l}\text { QTP- } \\
\text { EBT5014 }\end{array}$ \\
\hline$\square$ & mge & $\begin{array}{l}\text { Fagopyrum tataricum (L.) } \\
\text { Gaertn. }\end{array}$ & Polygonaceae & 47 & $\begin{array}{l}\text { QTP- } \\
\text { EBT5015 }\end{array}$ \\
\hline 吅 & ap yit & Malva rotundifolia Linn. & Malvaceae & 42 & $\begin{array}{l}\text { QTP- } \\
\text { EBT5016 }\end{array}$ \\
\hline 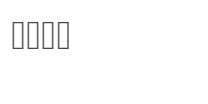 & $\begin{array}{l}\text { vot mop jyt } \\
\text { nyi }\end{array}$ & $\begin{array}{l}\text { Codonopsis pilosula (Franch.) } \\
\text { Nannf. }\end{array}$ & Campanulaceae & 41 & $\begin{array}{l}\text { QTP- } \\
\text { EBT5017 }\end{array}$ \\
\hline
\end{tabular}




\begin{tabular}{|c|c|c|c|c|c|}
\hline प्राप्रा & $\begin{array}{l}\text { mup sse } \\
\text { hnap bo }\end{array}$ & $\begin{array}{l}\text { Clinopodium urticifolium } \\
\text { (Hance) C. Y. Wu et Hsuan ex } \\
\text { H. W. Li }\end{array}$ & Lamiaceae & 40 & $\begin{array}{l}\text { QTP- } \\
\text { EBT5018 }\end{array}$ \\
\hline 口吅 & bbap zzip & Zanthoxylum.sp & Rutaceae & 37 & $\begin{array}{l}\text { QTP- } \\
\text { EBT5019 }\end{array}$ \\
\hline प्साप्रा & te $x y$ lat juo & Pyrola calliantha $\mathrm{H}$. Andr. & Ericaceae & 36 & $\begin{array}{l}\text { QTP- } \\
\text { EBT5020 }\end{array}$ \\
\hline 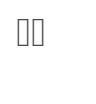 & mu ku & Litsea cubeba (Lour.) Pers. & Lauraceae & 35 & $\begin{array}{l}\text { QTP- } \\
\text { EBT5021 }\end{array}$ \\
\hline प्साप्रा & $\begin{array}{l}\text { gge bu a } \\
\text { nuo }\end{array}$ & $\begin{array}{l}\text { Gentiana rigescens Franch. ex } \\
\text { Hemsl. }\end{array}$ & Gentianaceae & 34 & $\begin{array}{l}\text { QTP- } \\
\text { EBT5022 }\end{array}$ \\
\hline प्साप्रा & $\begin{array}{l}\text { va hmip } \\
\text { syp hmip }\end{array}$ & Vaccinium $\mathrm{L}$. & Ericaceae & 33 & $\begin{array}{l}\text { QTP- } \\
\text { EBT5023 }\end{array}$ \\
\hline प्सा & $\begin{array}{l}\text { bbut qip } \\
\text { ddi }\end{array}$ & Sambucus adnata Wall. ex DC. & Caprifoliaceae & 32 & $\begin{array}{l}\text { QTP- } \\
\text { EBT5024 }\end{array}$ \\
\hline 口吅 & dur lap & Aconitum episcopale Leveille & Ranunculaceae & 32 & $\begin{array}{l}\text { QTP- } \\
\text { EBT5025 }\end{array}$ \\
\hline Q & ma & $\begin{array}{l}\text { Fargesia yunnanensis Hsueh } \\
\text { et Yi }\end{array}$ & Poaceae & 32 & $\begin{array}{l}\text { QTP- } \\
\text { EBT5026 }\end{array}$ \\
\hline वस्प & nip ho vo & $\begin{array}{l}\text { Schisandra lancifolia (Rehd. et } \\
\text { Wils.) A. C. Smith }\end{array}$ & Schisandraceae & 31 & $\begin{array}{l}\text { QTP- } \\
\text { EBT5027 }\end{array}$ \\
\hline 吅 & chyt jy & $\begin{array}{l}\text { Hypericum patulum Thunb. ex } \\
\text { Murray }\end{array}$ & Hypericaceae & 30 & $\begin{array}{l}\text { QTP- } \\
\text { EBT5028 }\end{array}$ \\
\hline 吅 & dda bbo & $\begin{array}{l}\text { Pteridium revolutum (BI.) } \\
\text { Nakai }\end{array}$ & Pteridiaceae & 30 & $\begin{array}{l}\text { QTP- } \\
\text { EBT5029 }\end{array}$ \\
\hline वस्पा & $\begin{array}{l}\text { vot mop } \\
\text { zza ke }\end{array}$ & $\begin{array}{l}\text { Taraxacum dasypodum V. } \\
\text { Soest }\end{array}$ & Compositae & 30 & $\begin{array}{l}\text { QTP- } \\
\text { EBT5030 }\end{array}$ \\
\hline प्साप्रा & $\begin{array}{l}\text { ddep bup a } \\
\text { tu }\end{array}$ & Urtica mairei Levl. & Urticaceae & 29 & $\begin{array}{l}\text { QTP- } \\
\text { EBT5031 }\end{array}$ \\
\hline 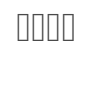 & $\begin{array}{l}\text { le rre bbut } \\
\text { cy }\end{array}$ & Paeonia delavayi Franch. & Paeoniaceae & 29 & $\begin{array}{l}\text { QTP- } \\
\text { EBT5032 }\end{array}$ \\
\hline 0 & fa xie yop & Lyonia ovalifolia (Wall.) Drude & Ericaceae & 25 & $\begin{array}{l}\text { QTP- } \\
\text { EBT5033 }\end{array}$ \\
\hline प & vup & Rubia podantha Diels & Rubiaceae & 24 & $\begin{array}{l}\text { QTP- } \\
\text { EBT5034 }\end{array}$ \\
\hline प्राप्र & $\begin{array}{l}\text { hnit nra a } \\
\text { hni }\end{array}$ & Chenopodium album L. & Chenopodiaceae & 23 & $\begin{array}{l}\text { QTP- } \\
\text { EBT5035 }\end{array}$ \\
\hline 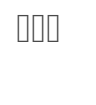 & $\begin{array}{l}\text { lop shet } \\
\text { map }\end{array}$ & Cimicifuga yunnanensis Hsiao & Ranunculaceae & 23 & $\begin{array}{l}\text { QTP- } \\
\text { EBT5036 }\end{array}$ \\
\hline 口吅 & te ssut & $\begin{array}{l}\text { Taxillus caloreas (Diels.) } \\
\text { Danser }\end{array}$ & Loranthaceae & 23 & $\begin{array}{l}\text { QTP- } \\
\text { EBT5037 }\end{array}$ \\
\hline
\end{tabular}




\begin{tabular}{|c|c|c|c|c|c|}
\hline 吅 & fut mop & Geranium strictipes & Geraniaceae & 21 & $\begin{array}{l}\text { QTP- } \\
\text { EBT5038 }\end{array}$ \\
\hline 吅 & te bbo & Pinus yunnanensis Franch. & Pinaceae & 21 & $\begin{array}{l}\text { QTP- } \\
\text { EBT5039 }\end{array}$ \\
\hline प्या & $\begin{array}{l}\text { a ddu sha } \\
\text { bbu }\end{array}$ & Anemone vitifolia Buch. -Ham. & Ranunculaceae & 20 & $\begin{array}{l}\text { QTP- } \\
\text { EBT5040 }\end{array}$ \\
\hline 吅 & nzy njip & $\begin{array}{l}\text { Rodgersia sambucifolia } \\
\text { Hemsl. }\end{array}$ & Saxifragaceae & 20 & $\begin{array}{l}\text { QTP- } \\
\text { EBT5041 }\end{array}$ \\
\hline 吅 & ba lat & Ehretia corylifolia C. H. Wright & Boraginaceae & 19 & $\begin{array}{l}\text { QTP- } \\
\text { EBT5042 }\end{array}$ \\
\hline प्राप्र & $\begin{array}{l}\text { ho bbo } \\
\text { ssut }\end{array}$ & Taxillus Tiegh.sp & Loranthaceae & 19 & $\begin{array}{l}\text { QTP- } \\
\text { EBT5043 }\end{array}$ \\
\hline प्या & $\begin{array}{l}\text { gep dep } \\
\text { map ma }\end{array}$ & Mahonia duclouxiana Gagn. & Berberidaceae & 18 & $\begin{array}{l}\text { QTP- } \\
\text { EBT5044 }\end{array}$ \\
\hline 吅 & xit zhup & Desmodium elegans DC. & Fabaceae & 18 & $\begin{array}{l}\text { QTP- } \\
\text { EBT5045 }\end{array}$ \\
\hline 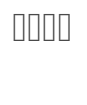 & $\begin{array}{l}\text { yo nyi ke } \\
\text { ddut }\end{array}$ & $\begin{array}{l}\text { Cynanchum otophyllum } \\
\text { Schneid. }\end{array}$ & Apocynaceae & 18 & $\begin{array}{l}\text { QTP- } \\
\text { EBT5046 }\end{array}$ \\
\hline 吅 & huo gat & Populus adenopoda Maxim & Salicaceae & 17 & $\begin{array}{l}\text { QTP- } \\
\text { EBT5047 }\end{array}$ \\
\hline प & hmup & Cannabis sativa $\mathrm{L}$. & Cannabaceae & 16 & $\begin{array}{l}\text { QTP- } \\
\text { EBT5048 }\end{array}$ \\
\hline प्या & $\begin{array}{l}\text { pat qi xy } \\
\text { hni }\end{array}$ & $\begin{array}{l}\text { Rumex nepalensis Spreng. var. } \\
\text { nepalensis }\end{array}$ & Polygonaceae & 16 & $\begin{array}{l}\text { QTP- } \\
\text { EBT5049 }\end{array}$ \\
\hline 吅 & yy ho & Salix matsudana Koidz. & Salicaceae & 16 & $\begin{array}{l}\text { QTP- } \\
\text { EBT5050 }\end{array}$ \\
\hline 啨 & ddut jy & $\begin{array}{l}\text { Aconitum carmichaelii } \\
\text { Debeaux }\end{array}$ & Ranunculaceae & 15 & $\begin{array}{l}\text { QTP- } \\
\text { EBT5051 }\end{array}$ \\
\hline 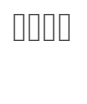 & $\begin{array}{l}\text { ddep bup a } \\
\text { nuop }\end{array}$ & $\begin{array}{l}\text { Girardinia diversifolia (Link) } \\
\text { Friis }\end{array}$ & Urticaceae & 14 & $\begin{array}{l}\text { QTP- } \\
\text { EBT5052 }\end{array}$ \\
\hline प्या & $\begin{array}{l}\text { gge jot hne } \\
\text { bbi }\end{array}$ & $\begin{array}{l}\text { Aristolochia griffithii Hook. f. } \\
\text { et Thoms. ex Duchartre }\end{array}$ & Aristolochiaceae & 14 & $\begin{array}{l}\text { QTP- } \\
\text { EBT5053 }\end{array}$ \\
\hline प्या & $\begin{array}{l}\text { hnit nra a } \\
\text { tu }\end{array}$ & Chenopodium album L. & Chenopodiaceae & 14 & $\begin{array}{l}\text { QTP- } \\
\text { EBT5054 }\end{array}$ \\
\hline प्या & sy lur qi py & $\begin{array}{l}\text { Cinnamomum tamala (Buch. - } \\
\text { Ham.) Th }\end{array}$ & Lauraceae & 14 & $\begin{array}{l}\text { QTP- } \\
\text { EBT5055 }\end{array}$ \\
\hline प्या & $\begin{array}{l}\text { va bu shy } \\
\text { ggo }\end{array}$ & Adenophora stricta Miq. & Campanulaceae & 13 & $\begin{array}{l}\text { QTP- } \\
\text { EBT5056 }\end{array}$ \\
\hline प्या & $\begin{array}{l}\text { yo sse la } \\
\text { bbo }\end{array}$ & Holboellia angustifolia Wallich & Lardizabalaceae & 13 & $\begin{array}{l}\text { QTP- } \\
\text { EBT5057 }\end{array}$ \\
\hline 些 & yyrx yyr & Ophiopogon Ker Gawl.sp & Asparagaceae & 13 & QTP- \\
\hline
\end{tabular}


EBT5058

\begin{tabular}{|c|c|c|c|c|c|}
\hline प्राप्रा & a mat lot si & $\begin{array}{l}\text { Serissa japonica (Thunb.) } \\
\text { Thunb. Nov. Gen. }\end{array}$ & Rubiaceae & 12 & $\begin{array}{l}\text { QTP- } \\
\text { EBT5059 }\end{array}$ \\
\hline प्र & gop gox & $\begin{array}{l}\text { Leontopodium calocephalum } \\
\text { (Franch.) Beauv. }\end{array}$ & Asteraceae & 12 & $\begin{array}{l}\text { QTP- } \\
\text { EBT5060 }\end{array}$ \\
\hline प्राप्रा & $\begin{array}{l}\text { li bbi syp } \\
\text { ddu }\end{array}$ & Cornus capitata & Cornaceae & 12 & $\begin{array}{l}\text { QTP- } \\
\text { EBT5061 }\end{array}$ \\
\hline 0 & $\begin{array}{l}\text { nyi mop } \\
\text { syp vo }\end{array}$ & $\begin{array}{l}\text { Vitis heyneana Roem. et } \\
\text { Schult. }\end{array}$ & Vitaceae & 12 & $\begin{array}{l}\text { QTP- } \\
\text { EBT5062 }\end{array}$ \\
\hline 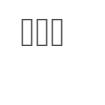 & $\begin{array}{l}\text { syp } \\
\text { ddat ssut }\end{array}$ & Taxillus Tiegh.sp & Loranthaceae & 12 & $\begin{array}{l}\text { QTP- } \\
\text { EBT5063 }\end{array}$ \\
\hline 0 & $\begin{array}{l}\text { a jji bbu } \\
\text { zza }\end{array}$ & Morus australis var. australis & Moraceae & 11 & $\begin{array}{l}\text { QTP- } \\
\text { EBT5064 }\end{array}$ \\
\hline $\mathrm{Qu}$ & ap zzit & Allium mairei Levl. & Amaryllidaceae & 11 & $\begin{array}{l}\text { QTP- } \\
\text { EBT5065 }\end{array}$ \\
\hline $\mathrm{s}$ & juo zy li & Ipomoea cairica (L.) Sweet & Convolvulaceae & 11 & $\begin{array}{l}\text { QTP- } \\
\text { EBT5066 }\end{array}$ \\
\hline 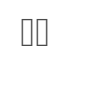 & mge vut & Illicium wardii A. C. Sm. & Magnoliaceae & 11 & $\begin{array}{l}\text { QTP- } \\
\text { EBT5067 }\end{array}$ \\
\hline 吅 & pat qi & Rumex acetosa $\mathrm{L}$. & Polygonaceae & 10 & $\begin{array}{l}\text { QTP- } \\
\text { EBT5068 }\end{array}$ \\
\hline प्र & syp bu & $\begin{array}{l}\text { Docynia delavayi (Franch.) } \\
\text { Schneid. }\end{array}$ & Rosaceae & 10 & $\begin{array}{l}\text { QTP- } \\
\text { EBT5069 }\end{array}$ \\
\hline प्राप्रा & $\begin{array}{l}\text { a mat nyuo } \\
\text { vut }\end{array}$ & $\begin{array}{l}\text { Cynoglossum amabile Stapf } \\
\text { et Drumm. }\end{array}$ & Boraginaceae & 9 & $\begin{array}{l}\text { QTP- } \\
\text { EBT5070 }\end{array}$ \\
\hline प्राप्रा & $\begin{array}{l}\text { bbu shy } \\
\text { ddut zza }\end{array}$ & $\begin{array}{l}\text { Arisaema erubescens (Wall.) } \\
\text { Schott }\end{array}$ & Araceae & 9 & $\begin{array}{l}\text { QTP- } \\
\text { EBT5071 }\end{array}$ \\
\hline 0 & jy sy & Coriaria nepalensis Wall. & Coriariaceae & 9 & $\begin{array}{l}\text { QTP- } \\
\text { EBT5072 }\end{array}$ \\
\hline $\mathrm{Qu}$ & vap ga & Rorippa indica (L.) Hiern & Brassicaceae & 9 & $\begin{array}{l}\text { QTP- } \\
\text { EBT5073 }\end{array}$ \\
\hline 吅 & vap ma & Brassica rapa L. & Brassicaceae & 9 & $\begin{array}{l}\text { QTP- } \\
\text { EBT5074 }\end{array}$ \\
\hline प्राप्रा & a zhat vop & Begonia grandis Dry. & Begoniaceae & 8 & $\begin{array}{l}\text { QTP- } \\
\text { EBT5075 }\end{array}$ \\
\hline प्र & ap jjit & $\begin{array}{l}\text { Pyracantha angustifolia } \\
\text { (Franch.) Schneid. }\end{array}$ & Rosaceae & 8 & $\begin{array}{l}\text { QTP- } \\
\text { EBT5076 }\end{array}$ \\
\hline प्र & bbie cy & Iridaceae nom. conserv. Sp & Iridaceae & 8 & $\begin{array}{l}\text { QTP- } \\
\text { EBT5077 }\end{array}$ \\
\hline प्राप्रा & $\begin{array}{l}\text { cep hlo a } \\
\text { hni }\end{array}$ & Fragaria vesca $\mathrm{L}$. & Rosaceae & 8 & $\begin{array}{l}\text { QTP- } \\
\text { EBT5078 }\end{array}$ \\
\hline
\end{tabular}




\begin{tabular}{|c|c|c|c|c|c|}
\hline 吅 & gop gox & $\begin{array}{l}\text { Leontopodium } \\
\text { leontopodioides (Willd.) } \\
\text { Beauv. }\end{array}$ & Asteraceae & 8 & $\begin{array}{l}\text { QTP- } \\
\text { EBT5079 }\end{array}$ \\
\hline प्राप्र & ho sha vu & $\begin{array}{l}\text { Fallopia multiflora (Thunb.) } \\
\text { Harald. }\end{array}$ & Polygonaceae & 8 & $\begin{array}{l}\text { QTP- } \\
\text { EBT5080 }\end{array}$ \\
\hline 吅 & rrup kot & $\begin{array}{l}\text { Cirsium lidjiangense Petrak ex } \\
\text { Hand. -Mazz. }\end{array}$ & Asteraceae & 8 & $\begin{array}{l}\text { QTP- } \\
\text { EBT5081 }\end{array}$ \\
\hline प्राप्र & vat bbu yo & $\begin{array}{l}\text { Incarvillea arguta (Royle) } \\
\text { Royle }\end{array}$ & Bignoniaceae & 8 & $\begin{array}{l}\text { QTP- } \\
\text { EBT5082 }\end{array}$ \\
\hline 吅 & bit map & Ricinus communis $\mathrm{L}$. & Euphorbiaceae & 7 & $\begin{array}{l}\text { QTP- } \\
\text { EBT5083 }\end{array}$ \\
\hline 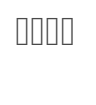 & $\begin{array}{l}\text { but fu zha } \\
\text { cy }\end{array}$ & Anemone rivularis Buch. -Ham. & Ranunculaceae & 7 & $\begin{array}{l}\text { QTP- } \\
\text { EBT5084 }\end{array}$ \\
\hline 吅 & it mup & Zea mays L. & Poaceae & 7 & $\begin{array}{l}\text { QTP- } \\
\text { EBT5085 }\end{array}$ \\
\hline प्राप्रा & $\begin{array}{l}\text { nyip ggu a } \\
\text { tu }\end{array}$ & $\begin{array}{l}\text { Lonicera trichosantha Bur. et } \\
\text { Franch. }\end{array}$ & Caprifoliaceae & 7 & $\begin{array}{l}\text { QTP- } \\
\text { EBT5086 }\end{array}$ \\
\hline प्राप्रा & $\begin{array}{l}\text { qy sse mge } \\
\text { hlop }\end{array}$ & $\begin{array}{l}\text { Fagopyrum } \\
\text { esculentum Moench }\end{array}$ & Polygonaceae & 7 & $\begin{array}{l}\text { QTP- } \\
\text { EBT5087 }\end{array}$ \\
\hline 吅 & syp ga & Prunus salicina Lindl. & Rosaceae & 7 & $\begin{array}{l}\text { QTP- } \\
\text { EBT5088 }\end{array}$ \\
\hline 吅 & va jy & Leycesteria formosa Wall. & Caprifoliaceae & 7 & $\begin{array}{l}\text { QTP- } \\
\text { EBT5089 }\end{array}$ \\
\hline प्राप्रा & $\begin{array}{l}\text { bbut xit ho } \\
\text { cy }\end{array}$ & $\begin{array}{l}\text { Agrimonia pilosa var. } \\
\text { nepalensis (D. Don) Nakai }\end{array}$ & Rosaceae & 6 & $\begin{array}{l}\text { QTP- } \\
\text { EBT5090 }\end{array}$ \\
\hline 口吅 & chyt jy & $\begin{array}{l}\text { Hypericum } \\
\text { forrestii (Chittenden) N. } \\
\text { Robson }\end{array}$ & Hypericaceae & 6 & $\begin{array}{l}\text { QTP- } \\
\text { EBT5091 }\end{array}$ \\
\hline प्सा & $\begin{array}{l}\text { gguo lyr } \\
\text { vop }\end{array}$ & $\begin{array}{l}\text { Psammosilene tunicoides W. } \\
\text { C. Wu et C. Y. Wu }\end{array}$ & Caryophyllaceae & 6 & $\begin{array}{l}\text { QTP- } \\
\text { EBT5092 }\end{array}$ \\
\hline प्राप्र & $\begin{array}{l}\text { gguo lyr } \\
\text { vop }\end{array}$ & Saponaria officinalis L. & Caryophyllaceae & 6 & $\begin{array}{l}\text { QTP- } \\
\text { EBT5093 }\end{array}$ \\
\hline 吅 & nyie lyt & $\begin{array}{l}\text { Ligusticum sinense } \\
\text { 'Chuanxiong' }\end{array}$ & Apiaceae & 6 & $\begin{array}{l}\text { QTP- } \\
\text { EBT5094 }\end{array}$ \\
\hline वस्ता & $\begin{array}{l}\text { gy sse mge } \\
\text { hlop }\end{array}$ & $\begin{array}{l}\text { Fagopyrum dibotrys (D. Don) } \\
\text { Hara }\end{array}$ & Polygonaceae & 6 & $\begin{array}{l}\text { QTP- } \\
\text { EBT5095 }\end{array}$ \\
\hline 吅 & za qip & Solanum tuberosum $\mathrm{L}$. & Solanaceae & 6 & $\begin{array}{l}\text { QTP- } \\
\text { EBT5096 }\end{array}$ \\
\hline प्साप्रा & $\begin{array}{l}\text { a ddu bba } \\
\text { jjo }\end{array}$ & $\begin{array}{l}\text { Notopterygium incisum Ting } \\
\text { ex H.T. Chang }\end{array}$ & Apiaceae & 5 & $\begin{array}{l}\text { QTP- } \\
\text { EBT5097 }\end{array}$ \\
\hline प्राप्र & bbu ga cy & Datura stramonium L. & Solanaceae & 5 & $\begin{array}{l}\text { QTP- } \\
\text { EBT5098 }\end{array}$ \\
\hline
\end{tabular}




\begin{tabular}{|c|c|c|c|c|c|}
\hline प्रा & bbut cha fu & $\begin{array}{l}\text { Bupleurum marginatum Wall. } \\
\text { ex DC. }\end{array}$ & Apiaceae & 5 & $\begin{array}{l}\text { QTP- } \\
\text { EBT5099 }\end{array}$ \\
\hline प्रा & bbut o jjie & Bidens pilosa $\mathrm{L}$. & Asteraceae & 5 & $\begin{array}{l}\text { QTP- } \\
\text { EBT5100 }\end{array}$ \\
\hline 吅 & di pu & $\begin{array}{l}\text { Pseudognaphalium } \\
\text { chrysocephalum Hilliard \& B. } \\
\text { L. Burtt }\end{array}$ & Caprifoliaceae & 5 & $\begin{array}{l}\text { QTP- } \\
\text { EBT5101 }\end{array}$ \\
\hline प्रा & gup sup bu & $\begin{array}{l}\text { Davallia trichomanoides } \\
\text { Blume }\end{array}$ & Davalliaceae & 5 & $\begin{array}{l}\text { QTP- } \\
\text { EBT5102 }\end{array}$ \\
\hline वस्ता & $\begin{array}{l}\text { hxie ggat } \\
\text { vat zza }\end{array}$ & Phytolacca acinosa Roxb. & $\begin{array}{l}\text { Phytolaccaceae } \\
\text { nom. conserv. }\end{array}$ & 5 & $\begin{array}{l}\text { QTP- } \\
\text { EBT5103 }\end{array}$ \\
\hline प्या & $\begin{array}{l}\text { hxie zy vap } \\
\text { ga }\end{array}$ & $\begin{array}{l}\text { Capsella bursa-pastoris (L.) } \\
\text { Medic. }\end{array}$ & $\begin{array}{l}\text { Cruciferae nom. } \\
\text { conserv. }\end{array}$ & 5 & $\begin{array}{l}\text { QTP- } \\
\text { EBT5104 }\end{array}$ \\
\hline प्राप्र & $\begin{array}{l}\text { lo ggur } \\
\text { bbar zziep }\end{array}$ & $\begin{array}{l}\text { Zanthoxylum } \\
\text { bungeanum Maxim. }\end{array}$ & Rutaceae & 5 & $\begin{array}{l}\text { QTP- } \\
\text { EBT5105 }\end{array}$ \\
\hline प्रा & ma bie cy & Verbena officinalis L. & Verbenaceae & 5 & $\begin{array}{l}\text { QTP- } \\
\text { EBT5106 }\end{array}$ \\
\hline प्या & $\begin{array}{l}\text { mop mo zy } \\
\text { ly }\end{array}$ & Elaeagnus umbellata Thunb. & Elaeagnaceae & 5 & $\begin{array}{l}\text { QTP- } \\
\text { EBT5107 }\end{array}$ \\
\hline 吅 & rrup kot & Cirsium shansiense Petrak & Asteraceae & 5 & $\begin{array}{l}\text { QTP- } \\
\text { EBT5108 }\end{array}$ \\
\hline 吅 & syp hmi & Juglans regia L. & Juglandaceae & 5 & $\begin{array}{l}\text { QTP- } \\
\text { EBT5109 }\end{array}$ \\
\hline 䏝 & syp vo & Amygdalus persica & Rosaceae & 5 & $\begin{array}{l}\text { QTP- } \\
\text { EBT5110 }\end{array}$ \\
\hline प्राप्र & $\begin{array}{l}\text { a nyie hxi } \\
\text { xy }\end{array}$ & Stellaria vestita Kurz & Caryophyllaceae & 4 & $\begin{array}{l}\text { QTP- } \\
\text { EBT5111 }\end{array}$ \\
\hline प्या & $\begin{array}{l}\text { bbu shy } \\
\text { cap hlo }\end{array}$ & $\begin{array}{l}\text { Duchesnea indica (Andr.) } \\
\text { Focke }\end{array}$ & Rosaceae & 4 & $\begin{array}{l}\text { QTP- } \\
\text { EBT5112 }\end{array}$ \\
\hline 吅 & chyt jy & $\begin{array}{l}\text { Hypericum acmosepalum N. } \\
\text { Robson }\end{array}$ & Hypericaceae & 4 & $\begin{array}{l}\text { QTP- } \\
\text { EBT5113 }\end{array}$ \\
\hline 吅 & chyt jy & Hypericum monogynum L. & Hypericaceae & 4 & $\begin{array}{l}\text { QTP- } \\
\text { EBT5114 }\end{array}$ \\
\hline प्रा & huo mop to & Clematis armandii Franch. & Ranunculaceae & 4 & $\begin{array}{l}\text { QTP- } \\
\text { EBT5115 }\end{array}$ \\
\hline 吅 & jy bbo & $\begin{array}{l}\text { Toxicodendron succedaneum } \\
\text { (L.) O. Kuntze }\end{array}$ & Anacardiaceae & 4 & $\begin{array}{l}\text { QTP- } \\
\text { EBT5116 }\end{array}$ \\
\hline 吅 & nyie lyt & Angelica L. sp & Apiaceae & 4 & $\begin{array}{l}\text { QTP- } \\
\text { EBT5117 }\end{array}$ \\
\hline 吅 & nyie lyt & Angelica likiangensis Wolff & Apiaceae & 4 & $\begin{array}{l}\text { QTP- } \\
\text { EBT5118 }\end{array}$ \\
\hline
\end{tabular}




\begin{tabular}{|c|c|c|c|c|c|}
\hline प्राप्रा & $\begin{array}{l}\text { shop shot } \\
\text { mop a nuo }\end{array}$ & Rubus niveus Thunb. & Rosaceae & 4 & $\begin{array}{l}\text { QTP- } \\
\text { EBT5119 }\end{array}$ \\
\hline प्राप्रा & $\begin{array}{l}\text { shuo ma a } \\
\text { hni }\end{array}$ & $\begin{array}{l}\text { Rhododendron delavayi } \\
\text { Franch. }\end{array}$ & Ericaceae & 4 & $\begin{array}{l}\text { QTP- } \\
\text { EBT5120 }\end{array}$ \\
\hline 吅 & sy zyt & $\begin{array}{l}\text { Lithocarpus cleistocarpus } \\
\text { (Seemen) Rehder et E. H. } \\
\text { Wilson }\end{array}$ & Fagaceae & 4 & $\begin{array}{l}\text { QTP- } \\
\text { EBT5121 }\end{array}$ \\
\hline प्से & vat mop ne & $\begin{array}{l}\text { Hedera nepalensis var. } \\
\text { sinensis (Tobl.) Rehd. }\end{array}$ & Araliaceae & 4 & $\begin{array}{l}\text { QTP- } \\
\text { EBT5122 }\end{array}$ \\
\hline 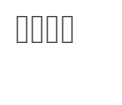 & $\begin{array}{l}\text { vot mop } \\
\text { rrup kot }\end{array}$ & Arctium lappa L. & Asteraceae & 4 & $\begin{array}{l}\text { QTP- } \\
\text { EBT5123 }\end{array}$ \\
\hline 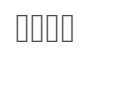 & $\begin{array}{l}\text { a ddu bba } \\
\text { jjo }\end{array}$ & Heracleum L. sp & Umbelliferae & 3 & $\begin{array}{l}\text { QTP- } \\
\text { EBT5124 }\end{array}$ \\
\hline 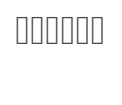 & $\begin{array}{l}\text { a ddu bba } \\
\text { jjo it zy }\end{array}$ & $\begin{array}{l}\text { Pimpinella candolleana Wight } \\
\text { et Arn. }\end{array}$ & Apiaceae & 3 & $\begin{array}{l}\text { QTP- } \\
\text { EBT5125 }\end{array}$ \\
\hline 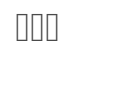 & $\begin{array}{l}\text { bbap } \\
\text { zzip ssut }\end{array}$ & Taxillus.sp & Loranthaceae & 3 & $\begin{array}{l}\text { QTP- } \\
\text { EBT5126 }\end{array}$ \\
\hline 吅 & bep bu & Asparagus filicinus D. Don & Asparagaceae & 3 & $\begin{array}{l}\text { QTP- } \\
\text { EBT5127 }\end{array}$ \\
\hline 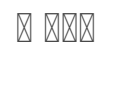 & $\begin{array}{l}\text { cep hlo a } \\
\text { hni }\end{array}$ & Fragaria gracilis Losinsk. & Rosaceae & 3 & $\begin{array}{l}\text { QTP- } \\
\text { EBT5128 }\end{array}$ \\
\hline प & dda & $\begin{array}{l}\text { Coniogramme intermedia } \\
\text { Hieron. }\end{array}$ & Pteridaceae & 3 & $\begin{array}{l}\text { QTP- } \\
\text { EBT5129 }\end{array}$ \\
\hline 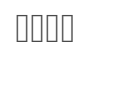 & $\begin{array}{l}\text { ddip sse ka } \\
\text { dda }\end{array}$ & $\begin{array}{l}\text { Hylotelephium spectabile } \\
\text { (Bor.) H. Ohba }\end{array}$ & Crassulaceae & 3 & $\begin{array}{l}\text { QTP- } \\
\text { EBT5130 }\end{array}$ \\
\hline 吅 & gge bu & Halenia elliptica D. Don & Gentianaceae & 3 & $\begin{array}{l}\text { QTP- } \\
\text { EBT5131 }\end{array}$ \\
\hline 吅 & ho bbo & Alnus nepalensis D. Don & Betulaceae & 3 & $\begin{array}{l}\text { QTP- } \\
\text { EBT5132 }\end{array}$ \\
\hline 吅 & jje bbo & $\begin{array}{l}\text { Cyclobalanopsis glauca } \\
\text { (Thunberg) Oersted }\end{array}$ & Fagaceae & 3 & $\begin{array}{l}\text { QTP- } \\
\text { EBT5133 }\end{array}$ \\
\hline 吅 & jop hop & $\begin{array}{l}\text { Incarvillea mairei (Lévl.) } \\
\text { Grierson }\end{array}$ & Bignoniaceae & 3 & $\begin{array}{l}\text { QTP- } \\
\text { EBT5134 }\end{array}$ \\
\hline 吅 & lie sy & Eucommia ulmoides Oliver & Eucommiaceae & 3 & $\begin{array}{l}\text { QTP- } \\
\text { EBT5135 }\end{array}$ \\
\hline 吅 & lop fip & Vicia amoena Fisch. & Papilionaceae & 3 & $\begin{array}{l}\text { QTP- } \\
\text { EBT5136 }\end{array}$ \\
\hline 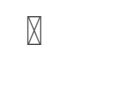 & ma & $\begin{array}{l}\text { Phyllostachys sulphurea } \\
\text { (Carr.) A. et C. Riv }\end{array}$ & Poaceae & 3 & $\begin{array}{l}\text { QTP- } \\
\text { EBT5137 }\end{array}$ \\
\hline 吅 & nbie cy & Iris wattii Baker & Iridaceae & 3 & $\begin{array}{l}\text { QTP- } \\
\text { EBT5138 }\end{array}$ \\
\hline
\end{tabular}




\begin{tabular}{|c|c|c|c|c|c|}
\hline$\nabla \otimes$ & pat qi & Rumex L. sp & Polygonaceae & 3 & $\begin{array}{l}\text { QTP- } \\
\text { EBT5139 }\end{array}$ \\
\hline 吅 & shox shot & Rubus pileatus Focke & Rosaceae & 3 & $\begin{array}{l}\text { QTP- } \\
\text { EBT5140 }\end{array}$ \\
\hline प्राप्रा & $\begin{array}{l}\text { shuo ma a } \\
\text { hni }\end{array}$ & $\begin{array}{l}\text { Rhododendron traillianum } \\
\text { Forrest et W. W. Smith. }\end{array}$ & Ericaceae & 3 & $\begin{array}{l}\text { QTP- } \\
\text { EBT5141 }\end{array}$ \\
\hline $\mathrm{Qut}$ & te shy jjix & Stellera chamaejasme L. & Thymelaeaceae & 3 & $\begin{array}{l}\text { QTP- } \\
\text { EBT5142 }\end{array}$ \\
\hline $\mathrm{Qut}$ & vie ap shy & $\begin{array}{l}\text { Pseudognaphalium } \\
\text { chrysocephalum Hilliard \& B. } \\
\text { L. Burtt }\end{array}$ & Asteraceae & 3 & $\begin{array}{l}\text { QTP- } \\
\text { EBT5143 }\end{array}$ \\
\hline प & vup & Rubia alata Roxb. & Rubiaceae & 3 & $\begin{array}{l}\text { QTP- } \\
\text { EBT5144 }\end{array}$ \\
\hline 吅 & yiep co & Galinsoga parviflora Cav. & Asteraceae & 3 & $\begin{array}{l}\text { QTP- } \\
\text { EBT5145 }\end{array}$ \\
\hline$\nabla \nabla$ & a hxa & Arisaema saxatile Buchet & Araceae & 2 & $\begin{array}{l}\text { QTP- } \\
\text { EBT5146 }\end{array}$ \\
\hline प्राप्रा & a jji lot gga & $\begin{array}{l}\text { Berchemia } \\
\text { yunnanensis Franch. }\end{array}$ & Rhamnaceae & 2 & $\begin{array}{l}\text { QTP- } \\
\text { EBT5147 }\end{array}$ \\
\hline 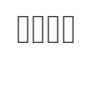 & $\begin{array}{l}\text { a mat lat } \\
\text { chu }\end{array}$ & Smilax ferox Wall. ex Kunth & Smilacaceae & 2 & $\begin{array}{l}\text { QTP- } \\
\text { EBT5148 }\end{array}$ \\
\hline प्राप्रा & a zhat xy si & $\begin{array}{l}\text { Polygonatum cirrhifolium } \\
\text { (Wall.) Royle }\end{array}$ & Asparagaceae & 2 & $\begin{array}{l}\text { QTP- } \\
\text { EBT5149 }\end{array}$ \\
\hline 吅 & bbop ddut & $\begin{array}{l}\text { Aconitum } \\
\text { carmichaelii Debeaux }\end{array}$ & Ranunculaceae & 2 & $\begin{array}{l}\text { QTP- } \\
\text { EBT5150 }\end{array}$ \\
\hline 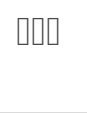 & bbut jjy yy & Prunella vulgaris $\mathrm{L}$. & Lamiaceae & 2 & $\begin{array}{l}\text { QTP- } \\
\text { EBT5151 }\end{array}$ \\
\hline प्राप्रा & $\begin{array}{l}\text { bbut tip xu } \\
\text { ge }\end{array}$ & $\begin{array}{l}\text { Campylotropis } \\
\text { hirtella (Franch.) Schindl. }\end{array}$ & Fabaceae & 2 & $\begin{array}{l}\text { QTP- } \\
\text { EBT5152 }\end{array}$ \\
\hline प्राप्रा & $\begin{array}{l}\text { hxie ggat } \\
\text { vat zza }\end{array}$ & $\begin{array}{l}\text { Colocasia esculenta (L.) } \\
\text { Schott. }\end{array}$ & Araceae & 2 & $\begin{array}{l}\text { QTP- } \\
\text { EBT5153 }\end{array}$ \\
\hline प्राप्रा & $\begin{array}{l}\text { lo ggur ap } \\
\text { jjit }\end{array}$ & Cotoneaster pannosus Franch. & Rosaceae & 2 & $\begin{array}{l}\text { QTP- } \\
\text { EBT5154 }\end{array}$ \\
\hline 吅 & $\begin{array}{l}\text { mgap } \\
\text { hniep }\end{array}$ & $\begin{array}{l}\text { Cerasus yunnanensis } \\
\text { (Franch.) Yü et Li }\end{array}$ & Rosaceae & 2 & $\begin{array}{l}\text { QTP- } \\
\text { EBT5155 }\end{array}$ \\
\hline 吅 & mu jjip & Acorus calamus $\mathrm{L}$. & Acoraceae & 2 & $\begin{array}{l}\text { QTP- } \\
\text { EBT5156 }\end{array}$ \\
\hline 口व & ry zot & Equisetum diffusum D. Don & Equisetaceae & 2 & $\begin{array}{l}\text { QTP- } \\
\text { EBT5157 }\end{array}$ \\
\hline प्याप्रा & $\begin{array}{l}\text { shuo ma a } \\
\text { ge }\end{array}$ & $\begin{array}{l}\text { Rhododendron decorum } \\
\text { Franch. }\end{array}$ & Ericaceae & 2 & $\begin{array}{l}\text { QTP- } \\
\text { EBT5158 }\end{array}$ \\
\hline
\end{tabular}




\begin{tabular}{|c|c|c|c|c|c|}
\hline प्राप्रा & $\begin{array}{l}\text { shuo ma } \\
\text { ma ge }\end{array}$ & $\begin{array}{l}\text { Rhododendron traillianum } \\
\text { Forrest et W. W. Smith. }\end{array}$ & Ericaceae & 2 & $\begin{array}{l}\text { QTP- } \\
\text { EBT5159 }\end{array}$ \\
\hline 㺃 & si six & Pinus armandii Franch. & $\begin{array}{l}\text { Pinaceae nom. } \\
\text { conserv. }\end{array}$ & 2 & $\begin{array}{l}\text { QTP- } \\
\text { EBT5160 }\end{array}$ \\
\hline 吅 & $\begin{array}{l}\text { ssup lot } \\
\text { juo }\end{array}$ & Keteleeria evelyniana Mast. & Pinaceae & 2 & $\begin{array}{l}\text { QTP- } \\
\text { EBT5161 }\end{array}$ \\
\hline वाप्रा & $\begin{array}{l}\text { ssup shut } \\
\text { hni }\end{array}$ & $\begin{array}{l}\text { Tsuga dumosa (D. Don) } \\
\text { Eichler in Engler u. Prantl }\end{array}$ & $\begin{array}{l}\text { Pinaceae nom. } \\
\text { conserv. }\end{array}$ & 2 & $\begin{array}{l}\text { QTP- } \\
\text { EBT5162 }\end{array}$ \\
\hline 吅 & syp yi & Armeniaca mume Sieb. & Rosaceae & 2 & $\begin{array}{l}\text { QTP- } \\
\text { EBT5163 }\end{array}$ \\
\hline वाप्रा & va vu gop & $\begin{array}{l}\text { Elsholtzia blanda (Benth.) } \\
\text { Benth }\end{array}$ & Lamiaceae & 2 & $\begin{array}{l}\text { QTP- } \\
\text { EBT5164 }\end{array}$ \\
\hline 吅 & vat dut & Pisum sativum $\mathrm{L}$. & Fabaceae & 2 & $\begin{array}{l}\text { QTP- } \\
\text { EBT5165 }\end{array}$ \\
\hline वस्पा & $\begin{array}{l}\text { vop qip } \\
\text { shop shot }\end{array}$ & Rubus sachalinensis Lévl. & Rosaceae & 2 & $\begin{array}{l}\text { QTP- } \\
\text { EBT5166 }\end{array}$ \\
\hline 吅 & vot hxit & Oxyria digyna (L.) Hill. & Polygonaceae & 2 & $\begin{array}{l}\text { QTP- } \\
\text { EBT5167 }\end{array}$ \\
\hline 吅 & vot nzy & $\begin{array}{l}\text { Debregeasia longifolia (Burm. } \\
\text { F.) Wedd. }\end{array}$ & Urticaceae & 2 & $\begin{array}{l}\text { QTP- } \\
\text { EBT5168 }\end{array}$ \\
\hline वस्पा & $\begin{array}{l}\text { ry ddu o } \\
\text { nuo }\end{array}$ & $\begin{array}{l}\text { Arundinella hookeri Munro ex } \\
\text { Keng }\end{array}$ & Poaceae & 2 & $\begin{array}{l}\text { QTP- } \\
\text { EBT5169 }\end{array}$ \\
\hline 吅 & $\mathrm{a} b \mathrm{bba}$ & $\begin{array}{l}\text { Dioscorea polystachya } \\
\text { Turczaninow }\end{array}$ & Dioscoreaceae & 1 & $\begin{array}{l}\text { QTP- } \\
\text { EBT5170 }\end{array}$ \\
\hline वस्पा & a hle va ry & Cyperus L. sp & Cyperaceae & 1 & $\begin{array}{l}\text { QTP- } \\
\text { EBT5171 }\end{array}$ \\
\hline वस्टा & a jji jie ddut & $\begin{array}{l}\text { Bauhinia brachycarpa Wall. ex } \\
\text { Benth. }\end{array}$ & Fabaceae & 1 & $\begin{array}{l}\text { QTP- } \\
\text { EBT5172 }\end{array}$ \\
\hline 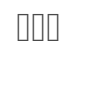 & a njy gop & $\begin{array}{l}\text { Lyonia compta (W. W. Smith et } \\
\text { Jeffr.) Hand. -Mazz. }\end{array}$ & Ericaceae & 1 & $\begin{array}{l}\text { QTP- } \\
\text { EBT5173 }\end{array}$ \\
\hline वस्पा & $\begin{array}{l}\text { a nyie bbyp } \\
\text { zy }\end{array}$ & $\begin{array}{l}\text { Cyclosorus dentatus (Forssk.) } \\
\text { Ching }\end{array}$ & Thelypteridaceae & 1 & $\begin{array}{l}\text { QTP- } \\
\text { EBT5174 }\end{array}$ \\
\hline वस्सा & $\begin{array}{l}\text { a nyie sip } \\
\text { sit }\end{array}$ & Rosa helenae Rehd. et Wils. & Rosaceae & 1 & $\begin{array}{l}\text { QTP- } \\
\text { EBT5175 }\end{array}$ \\
\hline वस्पा & $\begin{array}{l}\text { a nyut sy } \\
\text { tur }\end{array}$ & $\begin{array}{l}\text { Corylus yunnanensis (Franch.) } \\
\text { A. Camus }\end{array}$ & $\begin{array}{l}\text { Corylaceae nom. } \\
\text { conserv. }\end{array}$ & 1 & $\begin{array}{l}\text { QTP- } \\
\text { EBT5176 }\end{array}$ \\
\hline वस्पा & a zhat $x y$ si & $\begin{array}{l}\text { Polygonatum kingianum Coll. } \\
\text { et Hemsl. }\end{array}$ & Asparagaceae & 1 & $\begin{array}{l}\text { QTP- } \\
\text { EBT5177 }\end{array}$ \\
\hline 吅 & ba lat & Populus yunnanensis & Salicaceae & 1 & $\begin{array}{l}\text { QTP- } \\
\text { EBT5178 }\end{array}$ \\
\hline 吅 & ba ry & $\begin{array}{r}\text { Danthonia cumminsii J. D. } \\
\text { Page } 15 / 44\end{array}$ & Poaceae & 1 & QTP- \\
\hline
\end{tabular}




\begin{tabular}{|c|c|c|c|c|c|}
\hline 吅 & bba juo & $\begin{array}{l}\text { Musella lasiocarpa (Franchet) } \\
\text { C. Y. Wu ex H. W. Li }\end{array}$ & Musaceae & 1 & $\begin{array}{l}\text { QTP- } \\
\text { EBT5180 }\end{array}$ \\
\hline 吅 & bbit syp & Impatiens delavayi Franch. & Balsaminaceae & 1 & $\begin{array}{l}\text { QTP- } \\
\text { EBT5181 }\end{array}$ \\
\hline वस्प & bbu bbo di & Commelina communis Linn. & Commelinaceae & 1 & $\begin{array}{l}\text { QTP- } \\
\text { EBT5182 }\end{array}$ \\
\hline वस्पा & $\begin{array}{l}\text { bbut che ji } \\
\text { cy }\end{array}$ & $\begin{array}{l}\text { Lycopodium japonicum } \\
\text { Thunb. ex Murray }\end{array}$ & Lycopodiaceae & 1 & $\begin{array}{l}\text { QTP- } \\
\text { EBT5183 }\end{array}$ \\
\hline प्रा & bbut chy ni & $\begin{array}{l}\text { Ageratina adenophora } \\
\text { (Sprengel) R. M. King \& H. } \\
\text { Robinson }\end{array}$ & Asteraceae & 1 & $\begin{array}{l}\text { QTP- } \\
\text { EBT5184 }\end{array}$ \\
\hline 口吅 & bo hop & Mentha asiatica Boriss. -Bekrj. & Lamiaceae & 1 & $\begin{array}{l}\text { QTP- } \\
\text { EBT5185 }\end{array}$ \\
\hline वस्पा & $\begin{array}{l}\text { chyt sse la } \\
\text { ot }\end{array}$ & $\begin{array}{l}\text { Decaisnea insignis (Griffith) J. } \\
\text { D. Hooker et Thomson }\end{array}$ & Lardizabalaceae & 1 & $\begin{array}{l}\text { QTP- } \\
\text { EBT5186 }\end{array}$ \\
\hline वस्पा & $\begin{array}{l}\text { ddut bu o } \\
\text { hni }\end{array}$ & Pieris formosa (Wall.) D. Don & Ericaceae & 1 & $\begin{array}{l}\text { QTP- } \\
\text { EBT5187 }\end{array}$ \\
\hline वस्याप्रा & $\begin{array}{l}\text { die gu shut } \\
\text { ap mu }\end{array}$ & $\begin{array}{l}\text { Taxus yunnanensis W.C. } \\
\text { Cheng \& L.K. Fu }\end{array}$ & Taxaceae & 1 & $\begin{array}{l}\text { QTP- } \\
\text { EBT5188 }\end{array}$ \\
\hline 吅 & hly vo & Perilla frutescens (L.) Britt. & Lamiaceae & 1 & $\begin{array}{l}\text { QTP- } \\
\text { EBT5189 }\end{array}$ \\
\hline 吅 & hly vo & $\begin{array}{l}\text { Elsholtzia ciliata (Thunb.) } \\
\text { Hyland. }\end{array}$ & Lamiaceae & 1 & $\begin{array}{l}\text { QTP- } \\
\text { EBT5190 }\end{array}$ \\
\hline 口吅 & hxa cu & Schima argentea Pritz. & Theaceae & 1 & $\begin{array}{l}\text { QTP- } \\
\text { EBT5191 }\end{array}$ \\
\hline 吅 & hxa cu & $\begin{array}{l}\text { Ternstroemia gymnanthera } \\
\text { (Wight et Arn.) Beddome }\end{array}$ & Pentaphylacaceae & 1 & $\begin{array}{l}\text { QTP- } \\
\text { EBT5192 }\end{array}$ \\
\hline 吅 & jy sy & Coriaria terminalis & Coriariaceae & 1 & $\begin{array}{l}\text { QTP- } \\
\text { EBT5193 }\end{array}$ \\
\hline वस्पा & $\begin{array}{l}\text { lap bbo la } \\
\text { bbo }\end{array}$ & Holboellia latifolia Wall. & Lardizabalaceae & 1 & $\begin{array}{l}\text { QTP- } \\
\text { EBT5194 }\end{array}$ \\
\hline वस्पा & $\begin{array}{l}\text { lo ggur syp } \\
\text { ga }\end{array}$ & Rhamnus virgata Roxb. & Rhamnaceae & 1 & $\begin{array}{l}\text { QTP- } \\
\text { EBT5195 }\end{array}$ \\
\hline 吅 & ma mup & Galium spurium L. & Rubiaceae & 1 & $\begin{array}{l}\text { QTP- } \\
\text { EBT5196 }\end{array}$ \\
\hline 口吅 & mu hxit & Oxyria sinensis Hemsl. & Polygonaceae & 1 & $\begin{array}{l}\text { QTP- } \\
\text { EBT5197 }\end{array}$ \\
\hline वस्पा & $\begin{array}{l}\text { nyip ggu le } \\
\text { zhy }\end{array}$ & Celastrus stylosus Wall. & Celastraceae & 1 & $\begin{array}{l}\text { QTP- } \\
\text { EBT5198 }\end{array}$ \\
\hline \multirow[t]{2}{*}{ प्साप्रा } & \multirow[t]{2}{*}{ pat qi a tu } & Ligularia caloxantha (Diels) & \multirow[t]{2}{*}{ Asteraceae } & \multirow[t]{2}{*}{1} & \multirow[t]{2}{*}{ QTP- } \\
\hline & & Page 16/44 & & & \\
\hline
\end{tabular}




\begin{tabular}{|c|c|c|c|c|c|}
\hline प्रा & put nuop & Juncus effusus L. & Juncaceae & 1 & $\begin{array}{l}\text { QTP- } \\
\text { EBT5200 }\end{array}$ \\
\hline 0 & put nuop & $\begin{array}{l}\text { Juncus yunnanensis A. } \\
\text { Camus }\end{array}$ & Juncaceae & 1 & $\begin{array}{l}\text { QTP- } \\
\text { EBT5201 }\end{array}$ \\
\hline 0 & qi py & Curcuma longa L. & Zingiberaceae & 1 & $\begin{array}{l}\text { QTP- } \\
\text { EBT5202 }\end{array}$ \\
\hline प्र & rut shy & Quercus guyavifolia H. Léveillé & Fagaceae & 1 & $\begin{array}{l}\text { QTP- } \\
\text { EBT5203 }\end{array}$ \\
\hline 0 & rut shy & $\begin{array}{l}\text { Quercus aquifolioides Rehd. et } \\
\text { Wils. }\end{array}$ & Fagaceae & 1 & $\begin{array}{l}\text { QTP- } \\
\text { EBT5204 }\end{array}$ \\
\hline 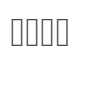 & $\begin{array}{l}\text { ryp ddu uo } \\
\text { nuo }\end{array}$ & $\begin{array}{l}\text { Arundinella hookeri Munro ex } \\
\text { Keng }\end{array}$ & Poaceae & 1 & $\begin{array}{l}\text { QTP- } \\
\text { EBT5205 }\end{array}$ \\
\hline प्र & shuo ma & Rhododendron.sp & Ericaceae & 1 & $\begin{array}{l}\text { QTP- } \\
\text { EBT5206 }\end{array}$ \\
\hline प्र & shuo ma & $\begin{array}{l}\text { Rhododendron simsii } \\
\text { Planch.sp }\end{array}$ & Ericaceae & 1 & $\begin{array}{l}\text { QTP- } \\
\text { EBT5207 }\end{array}$ \\
\hline 0 & shuo ma & $\begin{array}{l}\text { Rhododendron } \\
\text { simsii Planch.sp }\end{array}$ & Ericaceae & 1 & $\begin{array}{l}\text { QTP- } \\
\text { EBT5208 }\end{array}$ \\
\hline 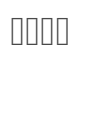 & $\begin{array}{l}\text { shuo ma } \\
\text { mgep zzyp }\end{array}$ & Fargesia yunnanensis & Ericaceae & 1 & $\begin{array}{l}\text { QTP- } \\
\text { EBT5209 }\end{array}$ \\
\hline प्र & shut bbo & Juniperus rigida Sieb. et Zucc. & Cupressaceae & 1 & $\begin{array}{l}\text { QTP- } \\
\text { EBT5210 }\end{array}$ \\
\hline 0 & shut bbo & Juniperus formosana Hayata & Cupressaceae & 1 & $\begin{array}{l}\text { QTP- } \\
\text { EBT5211 }\end{array}$ \\
\hline 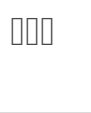 & sy a jjie & AcerL. sp & Sapindaceae & 1 & $\begin{array}{l}\text { QTP- } \\
\text { EBT5212 }\end{array}$ \\
\hline 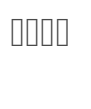 & syp bu a ge & Chaenomeles cathayensis & Rosaceae & 1 & $\begin{array}{l}\text { QTP- } \\
\text { EBT5213 }\end{array}$ \\
\hline $\mathrm{Qut}$ & syp bu ssut & Taxillus Tiegh.sp & Loranthaceae & 1 & $\begin{array}{l}\text { QTP- } \\
\text { EBT5214 }\end{array}$ \\
\hline प्र & syp nda & $\begin{array}{l}\text { Pyrus pyrifolia (Burm. F.) } \\
\text { Nakai }\end{array}$ & Rosaceae & 1 & $\begin{array}{l}\text { QTP- } \\
\text { EBT5215 }\end{array}$ \\
\hline 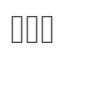 & syp vo ssut & Taxillus Tiegh.sp & Loranthaceae & 1 & $\begin{array}{l}\text { QTP- } \\
\text { EBT5216 }\end{array}$ \\
\hline 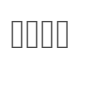 & $\begin{array}{l}\text { va bu syt } \\
\text { pup }\end{array}$ & Rosa sericea Lindl. & Rosaceae & 1 & $\begin{array}{l}\text { QTP- } \\
\text { EBT5217 }\end{array}$ \\
\hline 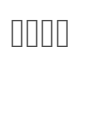 & $\begin{array}{l}\text { va bu syt } \\
\text { pup }\end{array}$ & Rosa sweginzowii Koehne & Rosaceae & 1 & $\begin{array}{l}\text { QTP- } \\
\text { EBT5218 }\end{array}$ \\
\hline 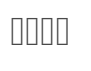 & vat ba sy & Parthenocissus semicordata & Vitaceae & 1 & QTP- \\
\hline
\end{tabular}




\begin{tabular}{|c|c|c|c|c|c|}
\hline प्राप्रा & $\begin{array}{l}\text { vot mop zy } \\
\text { ly }\end{array}$ & Solanum nigrum L. & Solanaceae & 1 & $\begin{array}{l}\text { QTP- } \\
\text { EBT5220 }\end{array}$ \\
\hline 吅 & yip syt & Coriandrum sativum & Apiaceae & 1 & $\begin{array}{l}\text { QTP- } \\
\text { EBT5221 }\end{array}$ \\
\hline प्राप्रा & yy zy ka py & Polygonum nepalense Meisn. & Polygonaceae & 1 & $\begin{array}{l}\text { QTP- } \\
\text { EBT5222 }\end{array}$ \\
\hline प्याप्रा & yy zy ka py & Persicaria (L.) Mill.sp & Polygonaceae & 1 & $\begin{array}{l}\text { QTP- } \\
\text { EBT5223 }\end{array}$ \\
\hline 吅 & zzit lyr & $\begin{array}{l}\text { Pistacia weinmanniifolia J. } \\
\text { Poisson ex Franchet }\end{array}$ & Anacardiaceae & 1 & $\begin{array}{l}\text { QTP- } \\
\text { EBT5224 }\end{array}$ \\
\hline 吅 & ma ke & Fargesia spathacea Franch. & Poaceae & 1 & $\begin{array}{l}\text { QTP- } \\
\text { EBT5225 }\end{array}$ \\
\hline प्राप्र & $\begin{array}{l}\text { cep hlo a } \\
\text { tu }\end{array}$ & $\begin{array}{l}\text { Fragaria nilgerrensis Schlecht. } \\
\text { ex Gay }\end{array}$ & Rosaceae & 1 & $\begin{array}{l}\text { QTP- } \\
\text { EBT5226 }\end{array}$ \\
\hline
\end{tabular}

\subsection{Folk nomenclature of plant species in the Xiaoliangshan Yi community}

Based on the plant names listed in Table 1, the folk nomenclature criteria for naming local plants used in the Yi ethnic community are based on the following (Figure 2): plant characteristics (127 species), cultural characteristics (91 species), usage (15 species) and plant habitat (11 species), and these are described in the following sub-sections.

\subsubsection{Plant names based on characteristics}

In this study, we documented 127 species with indigenous names that are based on plant characteristics. These species can be divided into four categories (although some species overlap categories), as follows: plant morphology (two types), plant taste, and plant scent. Of the 127 species, 99 names are based on plant morphology, and these are divided into two types: the first directly reflects the morphological characteristics of the plant and the second uses animal-related concepts and characteristics to describe the plant. In this second nomenclatural group, many of the plants have animal names (Table 3). Examples of plants in these categories are as follows: the locals use the term, $\otimes \otimes$ (Yi language phonetic name $₫ b b a j j o$ ), in the $Y i$ language for plants from the Umbelliferae family, which relates to the hollow stem of these plants; the Yi name for Bidens pilosa L. is $\triangle \otimes \square($ (bbut o jjie), which means "pitchforked-head grass"; and Anemone vitifolia Buch. -Ham., which is also known as wild cotton, is named $\mathrm{X} \otimes \mathrm{Q}$ ( $a$ ddu sha bbu), which relates the wool-like surface of the plant's achene to the hair of the fox. In addition, the leaf apexes of Polygonatum kingianum Coll. et Hemsl. and Polygonatum cirrhifolium (Wall.) Royle, which belong to the Polygonatum genus, are rolled downwards like a bird's claw, and these are named 畈 (a zhat xy si), which means "magpie's claws". Of the 127 plant 
species with names based on plant characteristics, 26 reflect the colour of the plant; for example, the Yi name for Pseudognaphalium chrysocephalum Hilliard \& B. L. Burtt is $\mathbb{Q} \otimes($ (vie ap shy), which means "yellow flower".

In addition, the names of nine species relate to the plant's taste. For example, Prunella vulgaris $\mathrm{L}$. is named $\mathrm{u}$ $\nabla$ (bbut jjy yy), which means "honey grass", and it is named in relation to the honey-like taste of its nectar. Furthermore, the Yi name for Begonia grandis Dry. is 畈区 (a zhat vop $j i)$, which means "magpie's sauerkraut", and it is so-named because of the sauerkraut-like taste of its stem.

Finally, one plant species is named based on its scent: Ageratina adenophora (Sprengel) R. M. King \& H. Robinson is named $\mathrm{X} \otimes($ (bbut chy $n i$ ), which means "stinky grass", because the whole plant has a distinctly unpleasant odour.

\section{Table 3}

Plant names based on animals in the Yi language. 
Animal Latin name

Fox

Notopterygium

incisum Ting ex H. T.

Chang

Fox

Pimpinella
candolleana Wight et

Arn.

Fox

Anemone

vitifolia Buch. -Ham.
Interpretation

language

phonetic

name
Voucher number a $\quad$ Q: "fox"+怄:"Hollow in stem" QTP-

ddu bba

jjo

प्राप्या

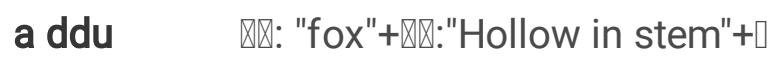

bba jjo it ख:"small"

QTP-

zy

वस्सा

a ddu

sha bbu

怄: "fox"+怄:"wool";

QTP-

EBT5004

a hle va

ry

प

邓:"rabbit"+邓:"chicken"+邓:"grass"

QTP-

EBT5005

Crow

Dipsacus

asper Wallich ex

Candolle

마맘

a jji bap

欧:"crow"

QTP-

mop

EBT5007

Crow

Morus australis var. australis

a jji bbu

zza

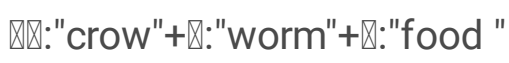

QTP-

EBT5008

Crow

Bauhinia

brachycarpa Wall. ex

प्राप्र

a jij jie

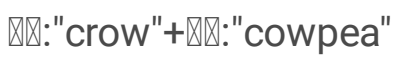

ddut

प्याप्रा

a jji lot

gga

yunnanensis Franch.

प्साप्त

Cyclosorus
dentatus (Forssk.)

Ching

Cat

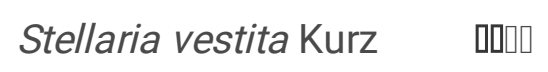

Cat

a nyie

bbyp zy

邓::"crow"+邓:"hand"

怄:"cat "

QTP-
EBT5015

QTP-

EBT5009

QTP-

EBT5010

QTP-

EBT5016

hxi xy

怄:"cat "

(B)

Cat Rosa helenae Rehd. et

Wils.

a nyie $\quad$ Q $:$ :"cat "

QTP-

sip sit

EBT5017

Monkey

Corylus

yunnanensis (Franch.)

A. Camus

Magpie Begonia grandis Dry.

Magpie Polygonatum

kingianum Coll. et

Hemsl.

a nyut sy 怄:"monkey "

tur

QTP-

EBT5018

\begin{tabular}{|c|c|c|c|c|c|}
\hline Magpie & $\begin{array}{l}\text { Polygonatum } \\
\text { cirrhifolium (Wall.) } \\
\text { Royle }\end{array}$ & प्याप्रा & $\begin{array}{l}\text { a zhat xy } \\
\text { si }\end{array}$ & 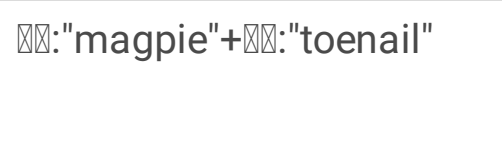 & $\begin{array}{l}\text { QTP- } \\
\text { EBT5021 }\end{array}$ \\
\hline Snake & $\begin{array}{l}\text { Duchesnea } \\
\text { indica (Andr.) Focke }\end{array}$ & स्साप्रा & $\begin{array}{l}\text { bbu shy } \\
\text { cap hlo }\end{array}$ & 怄:" snake"+怄:"strawberry" & $\begin{array}{l}\text { QTP- } \\
\text { EBT5037 }\end{array}$ \\
\hline
\end{tabular}




\begin{tabular}{|c|c|c|c|c|c|}
\hline Snake & $\begin{array}{l}\text { Arisaema } \\
\text { erubescens (Wall.) } \\
\text { Schott }\end{array}$ & प्साप्रा & $\begin{array}{l}\text { bbu } \\
\text { shy ddut } \\
\text { zza }\end{array}$ & $\begin{array}{l}\text { 讴:" snake"+怄:"Poisonous } \\
\text { food" }\end{array}$ & $\begin{array}{l}\text { QTP- } \\
\text { EBT5038 }\end{array}$ \\
\hline Goat & $\begin{array}{l}\text { Decaisnea } \\
\text { insignis (Griffith) J. D. } \\
\text { Hooker et Thomson }\end{array}$ & प्राप्रा & $\begin{array}{l}\text { chyt sse } \\
\text { la ot }\end{array}$ & 怄:"The goat cub" & $\begin{array}{l}\text { QTP- } \\
\text { EBT5060 }\end{array}$ \\
\hline Pig & $\begin{array}{l}\text { Colocasia } \\
\text { esculenta (L.) Schott. }\end{array}$ & प्राप्रा & $\begin{array}{l}\text { hxie ggat } \\
\text { vat zza }\end{array}$ & 怄:"Han Chinese"+怄:"Pig feed" & $\begin{array}{l}\text { QTP- } \\
\text { EBT5095 }\end{array}$ \\
\hline Pig & $\begin{array}{l}\text { Phytolacca } \\
\text { acinosa Roxb. }\end{array}$ & प्राप्रा & $\begin{array}{l}\text { hxie ggat } \\
\text { vat zza }\end{array}$ & 怄:"Han Chinese"+怄:"Pig feed" & $\begin{array}{l}\text { QTP- } \\
\text { EBT5096 }\end{array}$ \\
\hline Bird & $\begin{array}{l}\text { Capsella bursa- } \\
\text { pastoris (L.) Medic. }\end{array}$ & प्राप्रा & $\begin{array}{l}\text { hxie zy } \\
\text { vap ga }\end{array}$ & 怄:"bird" & $\begin{array}{l}\text { QTP- } \\
\text { EBT5097 }\end{array}$ \\
\hline Cattle & $\begin{array}{l}\text { Paeonia } \\
\text { delavayi Franch. }\end{array}$ & प्राप्रा & $\begin{array}{l}\text { le rre } \\
\text { bbut cy }\end{array}$ & 怄“cattle” & $\begin{array}{l}\text { QTP- } \\
\text { EBT5107 }\end{array}$ \\
\hline Foal & $\begin{array}{l}\text { Clinopodium } \\
\text { urticifolium (Hance) } \\
\text { C. Y. Wu et Hsuan ex } \\
\text { H. W. Li }\end{array}$ & प्याप्रा & $\begin{array}{l}\text { mup sse } \\
\text { hnap bo }\end{array}$ & 讴区foal"+"ear” & $\begin{array}{l}\text { QTP- } \\
\text { EBT5127 }\end{array}$ \\
\hline Cock & $\begin{array}{l}\text { Adenophora } \\
\text { stricta Miq. }\end{array}$ & प्साप्रा & $\begin{array}{l}\text { va bu } \\
\text { shy ggo }\end{array}$ & 怄:"cock" & $\begin{array}{l}\text { QTP- } \\
\text { EBT5186 }\end{array}$ \\
\hline Cock & Rosa sericea Lindl. & प्राप्रा & $\begin{array}{l}\text { va bu syt } \\
\text { pup }\end{array}$ & 怄:"cock" & $\begin{array}{l}\text { QTP- } \\
\text { EBT5187 }\end{array}$ \\
\hline Chicken & $\begin{array}{l}\text { Berberis } \\
\text { pruinosa Franch. }\end{array}$ & प्राप्र & $\begin{array}{l}\text { va ddot } \\
\text { chu }\end{array}$ & 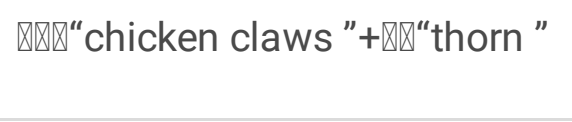 & $\begin{array}{l}\text { QTP- } \\
\text { EBT5190 }\end{array}$ \\
\hline Chicken & $\begin{array}{l}\text { Potentilla } \\
\text { lineata Treviranus }\end{array}$ & प्राप्रा & $\begin{array}{l}\text { va zza } \\
\text { hnap zzy }\end{array}$ & 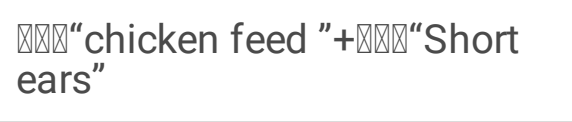 & $\begin{array}{l}\text { QTP- } \\
\text { EBT5194 }\end{array}$ \\
\hline Sow & Plantago major L. & प्याप्रा & $\begin{array}{l}\text { vot mop } \\
\text { ddie bbur }\end{array}$ & 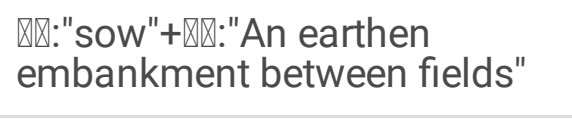 & $\begin{array}{l}\text { QTP- } \\
\text { EBT5206 }\end{array}$ \\
\hline Sow & $\begin{array}{l}\text { Codonopsis } \\
\text { pilosula (Franch.) } \\
\text { Nannf. }\end{array}$ & प्याप्रा & $\begin{array}{l}\text { vot mop } \\
\text { jyt nyi }\end{array}$ & 怄:"sow"+ष区: :"'" & $\begin{array}{l}\text { QTP- } \\
\text { EBT5207 }\end{array}$ \\
\hline Sow & Arctium lappa L. & प्याप्रा & $\begin{array}{l}\text { vot mop } \\
\text { rrup kot }\end{array}$ & 怄:"sow"+怄:"Cirsium" & $\begin{array}{l}\text { QTP- } \\
\text { EBT5208 }\end{array}$ \\
\hline Sow & Solanum nigrum L. & प्याप्रा & $\begin{array}{l}\text { vot mop } \\
\text { zy ly }\end{array}$ & 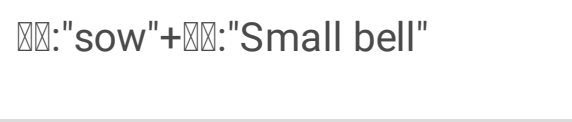 & $\begin{array}{l}\text { QTP- } \\
\text { EBT5209 }\end{array}$ \\
\hline Sow & $\begin{array}{l}\text { Taraxacum } \\
\text { dasypodum V. Soest }\end{array}$ & 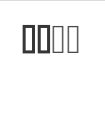 & $\begin{array}{l}\text { vot mop } \\
\text { zza ke }\end{array}$ & 怄:"sow"+邓:"food "+邓:"bitter" & $\begin{array}{l}\text { QTP- } \\
\text { EBT5210 }\end{array}$ \\
\hline
\end{tabular}

Note: Animal-related words in the Yi language and the Yi language phonetic name are shown in bold.

\subsubsection{Plant names based on habitat}


Many plant names in the Yi language are based on their native habitat (Table 4). Terms that describe the plant's habitat (such as the Yi word, 怄, which means "wild") are often used in the plant's name. For example,

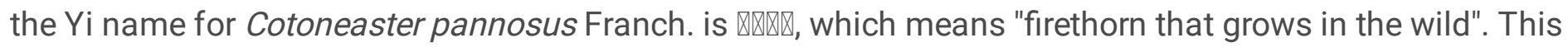
word distinguishes it from Pyracantha angustifolia (Franch.) Schneid., which is commonly planted around

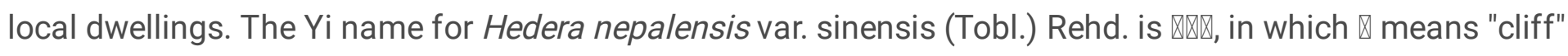
and $\mathrm{X}$ means "bead" because this plant is often found on cliff walls and it produces round bead-like fruit. Similarly, the names of many plants that generally grow near water or a swamp have the prefix “ $\square$ " or " $\mathbb{\text { Q }}$ ", which mean "water" and "swamp", respectively; for example, Polygonum nepalense Meisn. is named " $\nabla$ 㽖" in the Yi language and the willow tree is called " $\nabla \mathbb{Z}$ ".

Table 4

Plant names based on their habitat in the Yi language. 


\begin{tabular}{|c|c|c|c|c|}
\hline $\begin{array}{l}\text { Yi } \\
\text { language } \\
\text { name }\end{array}$ & $\begin{array}{l}\text { Yi language } \\
\text { phonetic name }\end{array}$ & Latin name & Interpretation & $\begin{array}{l}\text { Voucher } \\
\text { number }\end{array}$ \\
\hline व्या & lo ggur ap jjit & $\begin{array}{l}\text { Cotoneaster pannosus } \\
\text { Franch. }\end{array}$ & 讴: "In the wild" & $\begin{array}{l}\text { QTP- } \\
\text { EBT5110 }\end{array}$ \\
\hline प्या & $\begin{array}{l}\text { lo ggur bbar } \\
\text { zziep }\end{array}$ & $\begin{array}{l}\text { Zanthoxylum } \\
\text { bungeanum Maxim. }\end{array}$ & 呕: "In the wild" & $\begin{array}{l}\text { QTP- } \\
\text { EBT5111 }\end{array}$ \\
\hline वस्मा & lo ggur syp ga & Rhamnus virgata Roxb. & 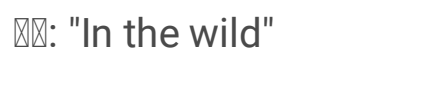 & $\begin{array}{l}\text { QTP- } \\
\text { EBT5112 }\end{array}$ \\
\hline वाप्रा & te shy jjix & Stellera chamaejasme L. & 怄:"By the pine roots" & $\begin{array}{l}\text { QTP- } \\
\text { EBT5183 }\end{array}$ \\
\hline ०स्सा & te $x y$ lat juo & Pyrola calliantha H. Andr. & 怄:"Under the pine tree" & $\begin{array}{l}\text { QTP- } \\
\text { EBT5185 }\end{array}$ \\
\hline 吅 & vat bbu yo & $\begin{array}{l}\text { Incarvillea arguta (Royle) } \\
\text { Royle }\end{array}$ & 凶:"The cliff" & $\begin{array}{l}\text { QTP- } \\
\text { EBT5199 }\end{array}$ \\
\hline प्राप्र & vat mop ne & $\begin{array}{l}\text { Hedera nepalensis var. } \\
\text { sinensis (Tobl.) Rehd. }\end{array}$ & 凶:"The cliff" & $\begin{array}{l}\text { QTP- } \\
\text { EBT5202 }\end{array}$ \\
\hline वर्पा & $\begin{array}{l}\text { vot mop ddie } \\
\text { bbur }\end{array}$ & Plantago major L. & $\begin{array}{l}\text { 怄:"An earthen } \\
\text { embankment between } \\
\text { fields" }\end{array}$ & $\begin{array}{l}\text { QTP- } \\
\text { EBT5206 }\end{array}$ \\
\hline$\square$ & yy ho & Salix matsudana Koidz. & 邓:"At the water's edge" & $\begin{array}{l}\text { QTP- } \\
\text { EBT5221 }\end{array}$ \\
\hline प्या & yy zy ka py & $\begin{array}{l}\text { Polygonum nepalense } \\
\text { Meisn. }\end{array}$ & $\begin{array}{l}\text { 怄:"At the edge of the } \\
\text { swamp" }\end{array}$ & $\begin{array}{l}\text { QTP- } \\
\text { EBT5222 }\end{array}$ \\
\hline व्या & yy zy ka py & Persicaria (L.) Mill.sp & $\begin{array}{l}\text { QW区:"At the edge of the } \\
\text { swamp" }\end{array}$ & $\begin{array}{l}\text { QTP- } \\
\text { EBT5223 }\end{array}$ \\
\hline$\square$ & za qip & Solanum tuberosum L. & 『:"In the soil" & $\begin{array}{l}\text { QTP- } \\
\text { EBT5225 }\end{array}$ \\
\hline
\end{tabular}

Note: Habitat-related words in the Yi language and the Yi language phonetic name are shown in bold in the table.

\subsubsection{Plant names based on culture}

Cultural heritage is another important element reflected in the folk botanical nomenclature of the Yi people. The influence of culture on the botanical nomenclature of the Yi community is reflected in the two types of names used: the first type is based on the Yi ethnic culture and the second is based on the combined effect of the Yi and Han cultures. Of the documented plants, the names of 71 species are based on the traditional

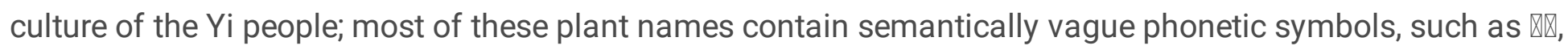

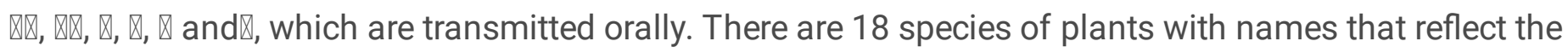
fusion between the traditional Yi culture and the Han culture, and most of these plants are of economic importance (Table 5). Of these, 11 are used for medicinal purposes, six are used as fodder, and one is used as 
food. Most of these plant names are derived from Chinese transliteration: some are direct transliterations of the Chinese name into the Yi language, and some have a Yi-language prefix added to a Chinese transliteration; for example, the folk name for Musella lasiocarpa (Franchet) C. Y. Wu ex H. W. Li and Musa basjoo Sieb. et Zucc. is $\mathbb{\text { Q }}$. This Yi name is romanised as "bba juo" which sounds like its Chinese name "ba

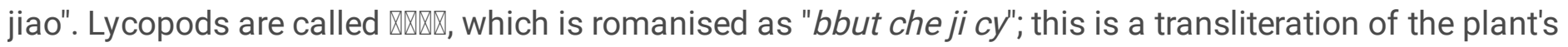
common Chinese name "choujin cao" with the prefix " $\nabla$ " added to indicate a herbaceous plant.

Table 5

Chinese loanwords in the folk plant names of the Yi community in Xiaoliangshan. 


\begin{tabular}{|c|c|c|c|c|c|}
\hline $\begin{array}{l}\text { Yi } \\
\text { language } \\
\text { name }\end{array}$ & $\begin{array}{l}\text { Yi language } \\
\text { phonetic } \\
\text { name }\end{array}$ & $\begin{array}{l}\text { Chinese name } \\
\text { (common } \\
\text { name) }\end{array}$ & $\begin{array}{l}\text { Chinese } \\
\text { phonetic name }\end{array}$ & Latin name & $\begin{array}{l}\text { Voucher } \\
\text { number }\end{array}$ \\
\hline 吅 & bba juo & 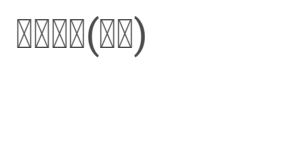 & $\begin{array}{l}\text { dì yǒng jīn lián( } \\
\text { bā jiāo) }\end{array}$ & $\begin{array}{l}\text { Musella lasiocarpa } \\
\text { (Franchet) C. Y. Wu ex H. } \\
\text { W. Li }\end{array}$ & $\begin{array}{l}\text { QTP- } \\
\text { EBT5028 }\end{array}$ \\
\hline प्रा & bbut cha fu & 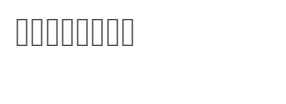 & $\begin{array}{l}\text { zhú yè chái hú } \\
\text { 『chái húð }\end{array}$ & $\begin{array}{l}\text { Bupleurum marginatum } \\
\text { Wall. ex DC. }\end{array}$ & $\begin{array}{l}\text { QTP- } \\
\text { EBT5039 }\end{array}$ \\
\hline प्राप्रा & $\begin{array}{l}\text { bbut che ji } \\
\text { cy }\end{array}$ & 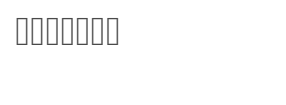 & $\begin{array}{l}\text { shí sōng ( chōu } \\
\text { jīn căo) }\end{array}$ & $\begin{array}{l}\text { Lycopodium japonicum } \\
\text { Thunb. ex Murray }\end{array}$ & $\begin{array}{l}\text { QTP- } \\
\text { EBT5040 }\end{array}$ \\
\hline प्राप्रा & $\begin{array}{l}\text { bbut tip xu } \\
\text { ge }\end{array}$ & 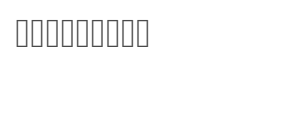 & $\begin{array}{l}\text { máo háng zi } \\
\text { shāo (tiě xuè } \\
\text { téng) }\end{array}$ & $\begin{array}{l}\text { Campylotropis } \\
\text { hirtella (Franch.) Schindl. }\end{array}$ & $\begin{array}{l}\text { QTP- } \\
\text { EBT5045 }\end{array}$ \\
\hline 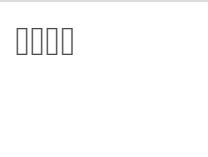 & $\begin{array}{l}\text { bbut xit ho } \\
\text { cy }\end{array}$ & 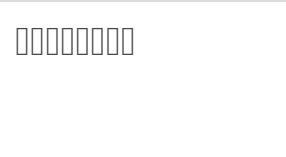 & $\begin{array}{l}\text { huáng lóng wěi } \\
\text { ( xiān hè cão ) }\end{array}$ & $\begin{array}{l}\text { Agrimonia pilosa var. } \\
\text { nepalensis (D. Don) } \\
\text { Nakai }\end{array}$ & $\begin{array}{l}\text { QTP- } \\
\text { EBT5046 }\end{array}$ \\
\hline 吅 & bep bu & 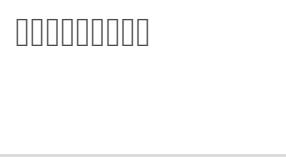 & $\begin{array}{l}\text { yáng chǐ tiān } \\
\text { mén dōng ( băi } \\
\text { bù ) }\end{array}$ & $\begin{array}{l}\text { Asparagus filicinus D. } \\
\text { Don }\end{array}$ & $\begin{array}{l}\text { QTP- } \\
\text { EBT5047 }\end{array}$ \\
\hline 吅 & bit map & 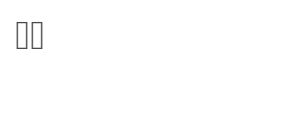 & bì má & Ricinus communis $\mathrm{L}$. & $\begin{array}{l}\text { QTP- } \\
\text { EBT5048 }\end{array}$ \\
\hline 吅 & bo hop & प्या & jiă bò he & $\begin{array}{l}\text { Mentha asiatica Boriss. - } \\
\text { Bekrj. }\end{array}$ & $\begin{array}{l}\text { QTP- } \\
\text { EBT5049 }\end{array}$ \\
\hline प्राप्रा & but fu zha cy & 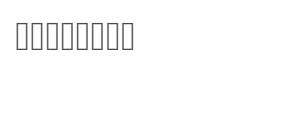 & $\begin{array}{l}\text { căo yù méi ( } \\
\text { hŭ zhăng căo ) }\end{array}$ & $\begin{array}{l}\text { Anemone rivularis Buch. } \\
\text {-Ham. }\end{array}$ & $\begin{array}{l}\text { QTP- } \\
\text { EBT5050 }\end{array}$ \\
\hline 吅 & dur lap & 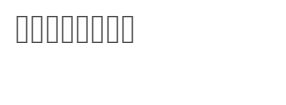 & $\begin{array}{l}\text { xī nán wū tóu ( } \\
\text { dǔ lă ) }\end{array}$ & $\begin{array}{l}\text { Aconitum } \\
\text { episcopale Leveille }\end{array}$ & $\begin{array}{l}\text { QTP- } \\
\text { EBT5070 }\end{array}$ \\
\hline प्रा & gup sup bu & प्य & gǔ suì bǔ & $\begin{array}{l}\text { Davallia trichomanoides } \\
\text { Blume }\end{array}$ & $\begin{array}{l}\text { QTP- } \\
\text { EBT5081 }\end{array}$ \\
\hline वस्प & ho sha vu & प्या & hé shǒu wū & $\begin{array}{l}\text { Fallopia multiflora } \\
\text { (Thunb.) Harald. }\end{array}$ & $\begin{array}{l}\text { QTP- } \\
\text { EBT5089 }\end{array}$ \\
\hline 吅 & it mup & 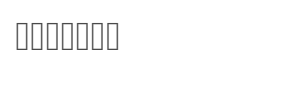 & $\begin{array}{l}\text { yù shǔ shǔ ( yù } \\
\text { mî) }\end{array}$ & Zea mays L. & $\begin{array}{l}\text { QTP- } \\
\text { EBT5098 }\end{array}$ \\
\hline 吅 & lop fip & 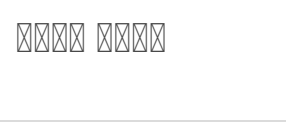 & $\begin{array}{l}\text { shān yě wān } \\
\text { dòu (lû́ féi ) }\end{array}$ & Vicia amoena Fisch. & $\begin{array}{l}\text { QTP- } \\
\text { EBT5113 }\end{array}$ \\
\hline प्स & lop shet map & प्साप्रा & $\begin{array}{l}\text { yún nán shēng } \\
\text { má }\end{array}$ & $\begin{array}{l}\text { Cimicifuga } \\
\text { yunnanensis Hsiao }\end{array}$ & $\begin{array}{l}\text { QTP- } \\
\text { EBT5114 }\end{array}$ \\
\hline वस्प & ma bie cy & प्राप्र & mă biān căo & Verbena officinalis L. & $\begin{array}{l}\text { QTP- } \\
\text { EBT5117 }\end{array}$ \\
\hline 吅 & vat dut & प्रा & wān dòu & Pisum sativum L. & $\begin{array}{l}\text { QTP- } \\
\text { EBT5200 }\end{array}$ \\
\hline \multirow[t]{2}{*}{ प्र } & yiep co & 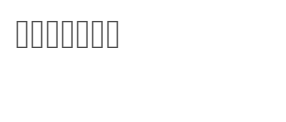 & $\begin{array}{l}\text { niú Xī jú ( yáng } \\
\text { câo ) }\end{array}$ & Galinsoga parviflora Cav. & $\begin{array}{l}\text { QTP- } \\
\text { EBT5217 }\end{array}$ \\
\hline & & & Page $25 / 44$ & & \\
\hline
\end{tabular}




\subsubsection{Plant names based on their common usage}

Naming plants based on their common usage is another method of nomenclature used by the Yi people in Xiaoliangshan (Table 6), and of the documented species, the names of 10 plants directly reflect their use. For example, Paeonia delavayi Franch., which is commonly used by the locals as strain-injury medication for

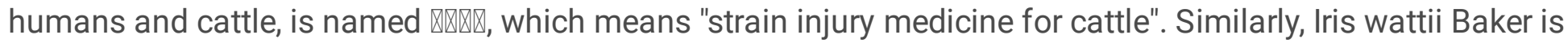
often used by the locals to treat pneumonia, and its Yi name is $\mathbb{}$, which means "pneumonia medicine".

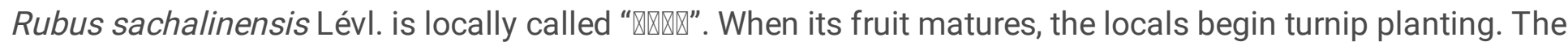
Yi term " $\triangle \mathbb{Q}$ " means "planting turnips"; therefore, the plant's name directly reflects its indicator function.

Table 6

Plant names based on their common use by the Yi community in Xiaoliangshan.

\begin{tabular}{|c|c|c|c|c|c|}
\hline $\begin{array}{l}\text { Yi } \\
\text { language } \\
\text { name }\end{array}$ & $\begin{array}{l}\text { Yi language } \\
\text { phonetic } \\
\text { name }\end{array}$ & Interpretation & Latin name & Family & $\begin{array}{l}\text { Voucher } \\
\text { number }\end{array}$ \\
\hline 吅 & bbit yop & $\begin{array}{l}\text { A medicine for } \\
\text { cracked skin }\end{array}$ & $\begin{array}{l}\text { Bulbophyllum } \\
\text { Thouars.sp }\end{array}$ & Orchidaceae & $\begin{array}{l}\text { QTP- } \\
\text { EBT5007 }\end{array}$ \\
\hline प्या & le rre bbut cy & $\begin{array}{l}\text { A medicine used to } \\
\text { cure tiredness of } \\
\text { cattle }\end{array}$ & $\begin{array}{l}\text { Paeonia } \\
\text { delavayi Franch. }\end{array}$ & Paeoniaceae & $\begin{array}{l}\text { QTP- } \\
\text { EBT5032 }\end{array}$ \\
\hline 吅 & bbu ga cy & $\begin{array}{l}\text { A medicine for } \\
\text { toothache }\end{array}$ & $\begin{array}{l}\text { Datura stramonium } \\
\text { L. }\end{array}$ & Solanaceae & $\begin{array}{l}\text { QTP- } \\
\text { EBT5098 }\end{array}$ \\
\hline 吅 & nbie cy & $\begin{array}{l}\text { A medicine for } \\
\text { pneumonia }\end{array}$ & Iris wattii Baker & Iridaceae & $\begin{array}{l}\text { QTP- } \\
\text { EBT5138 }\end{array}$ \\
\hline प्या & $\begin{array}{l}\text { vot mop zza } \\
\text { ke }\end{array}$ & Sow's feed & $\begin{array}{l}\text { Crepis lignea (Vaniot) } \\
\text { Babcock }\end{array}$ & Asteraceae & $\begin{array}{l}\text { QTP- } \\
\text { EBT5013 }\end{array}$ \\
\hline 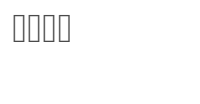 & $\begin{array}{l}\text { vot mop zza } \\
\text { ke }\end{array}$ & Sow's feed & $\begin{array}{l}\text { Taraxacum } \\
\text { dasypodum V. Soest }\end{array}$ & Compositae & $\begin{array}{l}\text { QTP- } \\
\text { EBT5030 }\end{array}$ \\
\hline प्या & a zhat vop ji & sauerkraut & Begonia grandis Dry. & Begoniaceae & $\begin{array}{l}\text { QTP- } \\
\text { EBT5075 }\end{array}$ \\
\hline प्या & te $x y$ lat juo & $\begin{array}{l}\text { A kind of tea that } \\
\text { grows under pine } \\
\text { trees }\end{array}$ & $\begin{array}{l}\text { Pyrola calliantha } \mathrm{H} \text {. } \\
\text { Andr. }\end{array}$ & Ericaceae & $\begin{array}{l}\text { QTP- } \\
\text { EBT5020 }\end{array}$ \\
\hline प्या & $\begin{array}{l}\text { vop qip shop } \\
\text { shot }\end{array}$ & Planting rutabaga & $\begin{array}{l}\text { Rubus } \\
\text { sachalinensis Lévl. }\end{array}$ & Rosaceae & $\begin{array}{l}\text { QTP- } \\
\text { EBT5166 }\end{array}$ \\
\hline प्या & a nyut sy tur & $\begin{array}{l}\text { Used for making } \\
\text { chisel handles }\end{array}$ & $\begin{array}{l}\text { Corylus yunnanensis } \\
\text { (Franch.) A. Camus }\end{array}$ & $\begin{array}{l}\text { Corylaceae } \\
\text { nom. } \\
\text { conserv. }\end{array}$ & $\begin{array}{l}\text { QTP- } \\
\text { EBT5176 }\end{array}$ \\
\hline
\end{tabular}




\subsection{Analysis of the basic structure of traditional plant names of the Yi people in Xiaoliangshan}

In the folk nomenclature system of the Yi people in Xiaoliangshan, plant names have a binomial or nonbinomial structure (Figure 3). A binomial folk plant name consists of two Yi words: one of these is the core or the primary name and the other is a modifier used to describe or clarify the core word. A non-binomial plant name consists of one Yi word. Of the local plants documented in this study, 67 species have binomial names and 161 have non-binomial names. The following examples show the binomial structure of folk botanical names in the Xiaoliangshan ethnic community, where a modifier is added to the core word to highlight its characteristics.:

\section{Example 1}

Latin name: Ageratina adenophora (Sprengel) R. M. King \& H. Robinson

Yi name: $\otimes($ core word) $+\mathbb{\nabla}$ (modifier)

Meaning: smelly (modifier) + (core word)

Example 2

Latin name: Rhododendron decorum Franch.

Yi name: $\mathbb{Q}$ (core word) $+\mathbb{Q}($ (modifier)

Meaning: Big (modifier) + Azalea (core word)

Plant names with a non-binomial structure consist of one semantically ambiguous core word or a Chinese

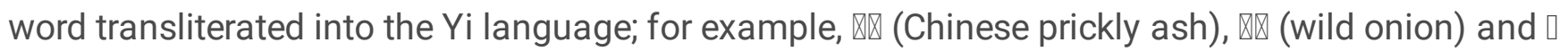
$\otimes$ (Asparagus filicinus).

\subsection{Correspondence between plant names and species in the folk nomenclature of the Yi people in Xiaoliangshan}

This study found that not all folk plant names and taxonomic species have a one-to-one correspondence; some plant species have multiple folk names, and one folk name may be used for multiple species (Figure 4). The name to species correspondence is elucidated as follows:

(1)One folk plant name corresponds to one species. Of the folk names, 171 correspond to one plant species;

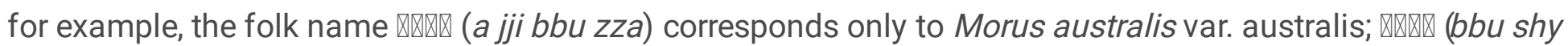

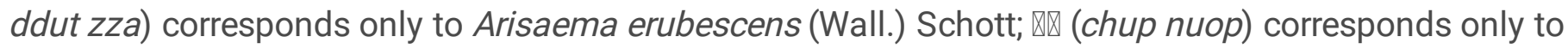

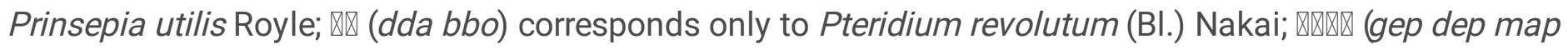
ma) corresponds only to Mahonia duclouxiana Gagn.; 怄 (huo gat) corresponds only to Populus adenopoda 
Maxim; 怄 (jy bbo) corresponds only to Toxicodendron succedaneum (L.) O. Kuntze; corresponds only to Cornus capitata and $\mathrm{X} \mathrm{Q}(\mathrm{mu} \mathrm{ku}$ ) corresponds only to Litsea cubeba (Lour.) Pers..

(2) Two folk names corresponding to one plant species. Of the plant names, four have two folk names corresponding to one scientific name. Chenopodium album $\mathrm{L}$. is an edible wild plant commonly used by locals for food and fodder. As the locals classify it as two different plants, it has two different Yi names:

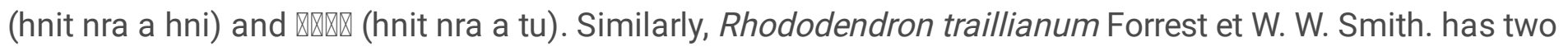

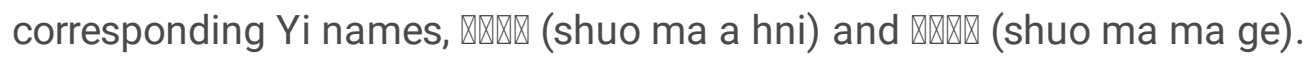

(3) One folk name corresponding to multiple plant species. Of the plant names, 53 have folk plant names that correspond to multiple plant species. For example, four different plant species correspond to the Yi name $\mathrm{au}$ (chyt jy): Hypericum acmosepalum N. Robson, Hypericum monogynum L., Hypericum forrestii (Chittenden) N. Robson, and Hypericum patulum Thunb. x Murray; two different species correspond to the Yi name $\mathbb{\text { Q }}$ (jy sy): Coriaria terminalis and Coriaria nepalensis Wall.; and two different species correspond to the Yi name (shut bbo): Juniperus rigida Sieb. et Zucc. and Juniperus formosana Hayata.

\subsection{Comparison between folk botanical nomenclature of the Yi people in Xiaoliangshan and the Yi people in the Daliangshan}

We compared the folk botanical nomenclature of the Yi people in the Daliangshan [3] with that of the Yi community in Xiaoliangshan (Figure 5) and found that the plant names and usages of the Yi people in the two places overlapped to a certain extent. More specifically, the two places have 55 plant names in common (Figure $5 \mathrm{~A}$ ), corresponding to approximately $24 \%$ of the total number of plant names collected in Xiaoliangshan. However, only 18 out of the 55 names represent the same species in both places and the remaining names represent different species. Most of these 18 identical plant species have been used by the

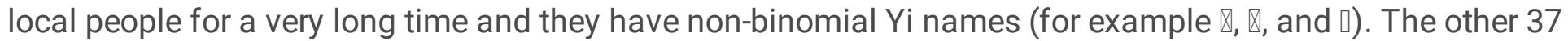
plant names that are common to both places refer to different plants; however, the plants belong to the same family or genus in modern taxonomy, or they have some similar attributes. For example, the Yi name $\mathbb{Q} \mathbb{Q} \mathrm{Q}$ is used for three species of the Pinaceae family: in the Daliangshan it refers to Abies fabri (Mast.) Craib and Larix potaninii Batalin, whereas in Xiaoliangshan it refers to Tsuga dumosa (D. Don) Eichler in Engler u. Prantl. In addition, the Yi name $\mathbb{Q}$ represents three different species of the Artemisia genus: Artemisia annua

L. and another species of wormwood in the Daliangshan, and Artemisia indica Willd. in Xiaoliangshan. In the

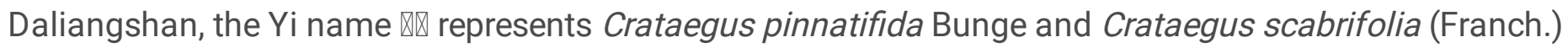
Rehder, whereas in Xiaoliangshan, it refers to Docynia delavayi (Franch.) Schneid.. Similarly, in the Daliangshan, the Yi word, 怄, refers to Populus sp. L., whereas in Xiaoliangshan, it refers to Populus yunnanensis and Ehretia corylifolia C. H. Wright.

\section{Discussion}




\section{4 .1 Characteristics of folk plant nomenclature of the Yi people in Xiaoliangshan}

Many ethnic groups name plant species based on what the plant resembles [2,9,33, 34]. This method reflects a direct approach of recognising plants through the human senses, and it is based on the plants' visual appearance and taste. All such information is contained in the indigenous plant name. Similarly, the Yi people in Xiaoliangshan named plants based on their characteristics, and the names are often related to the characteristic shape, colour, smell, or taste of the plant. In addition to directly describing plant characteristics, the folk plant names used by the Xiaoliangshan Yi people are often based on animals, a nomenclature practice that is common in other places [3,35, 36,37]. The frequent use of animal names for plants can be explained by the traditional livelihood of the Yi people, which involves various animals. Some studies have reported that to adapt to the demands of the mountainous environment in southwestern China, the Yi people formed a lifestyle based on farming and pastoral practices, and their dependence on livestock has thus been relatively high $[22,38]$. It is therefore likely that when naming local plants, some of the salient features of a plant would stimulate a certain sensory response in the observer and cause them to associate the plant with a familiar object, which was ultimately used in the name selected for the plant. Due to the semi-pastoral lifestyle of the Yi people, it would be easy for an observer to assign suitable animal characteristics to a plant and use them to describe the plant, which is a nomenclature practice similar to that of the Mongolian herders [36].

Of the indigenous plant names of the Yi people in Xiaoliangshan, many include words that describe the plant's habitat, such as water, swamp, and field. This practice is also common in the folk plant nomenclature of the Mongolian and the Tung ethnic groups $[39,40]$. Adding a habitat-related word to the plant's name would likely help distinguish it from other species and make it easier to find and collect. For example, the $\mathrm{Yi}$ people believe that Rhamnus virgata Roxb. is a wild plum (Prunus salicina Lindl.), so they use a habitatbased name to reflect the similarities and the differences between the two plant species. Another example is Plantago major L., which is a type of plant often used by the locals as pig feed; the Yi name of this plant reflects its habitat, which provides a clue to locals about where to find it.

Folk plant nomenclature is also based on oral traditions [41-43]. This study found that many plant names that are transmitted orally often contain semantically ambiguous phonetic symbols, and this finding is consistent with those of other studies of folk plant names used in traditional rituals within this area [14]. It is believed that in this type of nomenclature, in which the plant name is assigned directly and passed on orally, the unique name refers to the biological organism itself, and no further semantic analysis is therefore required.

In addition to the local Yi culture, the Han culture has also impacted the folk nomenclature of the Yi community in Xiaoliangshan. Many foreign plants have been introduced to the community, and the majority are used for medicinal purposes. The Yi people either directly transliterate the Chinese names of the introduced plants or add a Yi-language prefix to the Chinese transliteration. It is believed that these Chinese loanwords were introduced to Xiaoliangshan in a brief window of time during the 1960s when there was a lack of medical care in China, and the "barefoot doctor" policy was implemented [44]. The local government 
conducted basic medical training for barefoot doctors lasting four to six months [45], and they were later employed in local villages as healthcare providers, which may have helped spread knowledge about Chinese herbal medicine in the Xiaoliangshan area. The increased use of borrowed Chinese names may also be related to the popularisation of standard Mandarin in basic education, ethnic integration, and the transformation of traditional lifestyles in the Xiaoliangshan region, and this was determined by another study based on the folk botanical nomenclature of the Yi people in Daliangshan [3].

With respect to the function-based plant nomenclature of the Yi people in Xiaoliangshan, the indigenous names employed reflect the plant's use or its value to humans and animals. This is similar to the functionbased plant names used by the Han ethnic group [46]. For example, the Yi name of Paeonia delavayi Franch., which is used by the locals to treat injuries in humans and cattle, directly reflects the plant's use. It is believed that this practice is also related to the traditional livelihood of the Yi people. The Yi people are nomadic farmers, and cattle are the main source of power used in their traditional farming practices [47]. As wasteland reclamation is labour intensive, both humans and animals, but especially cattle, would often suffer strain injuries. Therefore, the plant that was used as a folk remedy for strain injuries has been given an indigenous name that reflects this use. Similarly, the Yi name for Rubus sachalinensis Lévl. reflects its indicator plant function. The Yi people in Xiaoliangshan have a long-standing practice of turnip cultivation, and this overwintering vegetable is sown seasonally and continues to be a staple food of the Yi people [48]. However, turnips are formed approximately three months after flowering $[49,50]$, and such a short growth cycle means that locals need to correctly assess the optimum sowing time for the crop. The fruiting period of Rubus sachalinensis Lévl. is from August to September [51], which coincides with the time when the locals begin turnip planting. Therefore, the indigenous name for Rubus sachalinensis Lévl. reflects this indicator plant's function of notifying the Yi people that it is time to sow turnips.

This study found that the Yi people named useful plants using a binomial and non-binomial structure. This is consistent with the findings of a study focusing on plants used in religious rituals [14]. The binomial structure for the botanical nomenclature used by the Yi people is similar to that of the Dai and Han ethnic groups $[9,52]$. It is believed that this naming structure is used due to practical considerations: it enables the locals to learn important information about different plants, including their life form, habitat, and functions, which ultimately makes it easier to recognise and remember useful plants. The non-binomial names tend to reflect the characteristics of the local language; these names are generally transmitted orally using semantically ambiguous phonetic symbols. Plants such as Fagopyrum tataricum (L.) Gaertn., Cannabis sativa L., and Oryza sativa L. have been cultivated by the Yi people for a very long time [53-55], and the ancient Yi names of these plants have a monosyllabic no-binomial structure. They are often used as root words when naming more complex plants, which indicates their important roles in the lives of the local Yi people [56].

This study found that there were three types of correspondence between plant names used by the Yi people in Xiaoliangshan and the plant species, namely: one plant name for one plant species, two plant names for one plant species, and one plant name for multiple plant species. These correspondence types are similar to those found by Raven et al., who studied the folk nomenclature and taxonomy of indigenous plants in Mexico [4], and to those of the Chinese Mongolian ethnic group [36]. Investigating the correspondence between folk plant names and plant species enables us to better understand how the Yi people in

Page 30/44 
Xiaoliangshan perceive and recognise plants. This is especially true when multiple indigenous names are given to one plant species, or when one indigenous name corresponds to multiple plant species. For example, the locals classify Chenopodium album L. as two plants, which is reflected by the folk nomenclature. Both

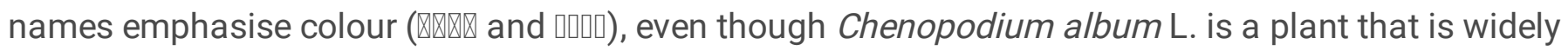
distributed and has many morphological variations [57]. Another example is that four different species of Hypericum are all named $\mathbb{\Delta}$ in the Yi language. These species are primarily found in southwestern China [58], and they are all important medicinal plants used in Xiaoliangshan to treat the same health problem. It is thus believed that they share one indigenous name in Xiaoliangshan because they have a similar form and function.

\subsection{The cultural connotations of the folk plant nomenclature of the Yi people in Xiaoliangshan}

With respect to the relationship between culture and ethnobotanical knowledge, ethnobotanists Cassandra L. Quave and Andrea Pieroni stated that regardless of the living environment, the decisions and behaviour of an ethnic group of people are influenced by their culture [59]. Therefore, ethnobotanical knowledge can reveal how the same ethnic group living in different geographical locations interacts with the environment. The Yi people in Xiaoliangshan and those in the Daliangshan belong to the same ethnic group, but their living environments differ. Therefore, comparing the folk names of the plant species used by the two groups of Yi people can enhance our understanding of how the Yi language evolved to provide a basis for selecting appropriate measures to protect traditional knowledge.

In this study, we found the Yi people in the Xiaoliangshan, and the Daliangshan refer to different plant species that correspond to the same indigenous name and plant species that have different indigenous names. It was found that 37 indigenous plant names refer to different plants in the two places, but all these plants belong to the same family or genus (Table 1), or they have certain modern taxonomic similarities. It is considered that this phenomenon may be related to the following circumstances: for plants belonging to the same genus or family, such as fir, redwood, and hemlock, it is believed that when the Yi people, who migrated from the Daliangshan to the unfamiliar environment of Xiaoliangshan, encountered a plant that resembled a familiar species, the newly discovered plant was given the same name as the plant it resembled. For example, it is believed that Crataegus pinnatifida Bge., Crataegus scabrifolia (Franch.) Rehd., and Docynia delavayi (Franch.) Schneid. have all been given the same name because they all produce sour-tasting fruit. In addition, Chaenomeles cathayensis is called $\mathbb{X} \mathbb{X}$ in the Yi language and it also has sour fruit; the main part of its indigenous name indicates that the Xiaoliangshan Yi people classified it as the same species as the other three plants. In summary, it is believed that the Yi people living in the two different environments use the same indigenous name for different plant species because the plants were named based on a common characteristic by those who migrated to Xiaoliangshan.

In addition, the study found that there are 41 plant species in common between the two areas: 27 have identical indigenous names and 14 have different names. Among the 14 species with different names, the folk names of Eucommia ulmoides Oliv., Taraxacum mongolicum Hand. -Mazz. and Aristolochia debilis

Page $31 / 44$ 
Siebold \& Zucc used by the Yi community in the Daliangshan are Chinese loanwords, whereas the indigenous names of these plants are retained in Xiaoliangshan. This could be attributed to the fact that the Yi people in Xiaoliangshan are still using traditional plant knowledge, and the indigenous names of these plants have thus been preserved despite the introduction of Han culture into the community. For example, the boiled root of Eucommia ulmoides Oliv. is used to treat bruises and backaches, dandelion is used to reduce inflammation and clear away toxins and Aristolochia is often used to treat stomach ailments and for deworming. All these plants are easily accessible local resources. In contrast, ethnobotanical research conducted in the Daliangshan demonstrated that the folk nomenclature of plant species in this region has been largely replaced by that of the Han ethnic group [3]. These findings provide valuable insights for the protection of ethnobotanical knowledge; the continuous practice of ethnobotanical knowledge based on existing records may greatly contribute to its preservation.

\subsection{The significance of the plant nomenclature of the Yi ethnic group in Xiaoliangshan}

Hengduan Mountains is a global diversity hotspot [60], but China's accelerated urbanisation progress has resulted in a severe loss of biodiversity within this region [61]. To protect biodiversity more effectively in ethnic minority areas, it is necessary to first preserve cultural diversity, and particularly to protect aspects of ethnic cultures that are closely related to biodiversity. The folk nomenclature of fauna and flora are important parts of cultural diversity and are essential for use in biodiversity conservation [62]. This is reflected primarily in the following two aspects: first, from a local perspective, folk nomenclature reflects an indigenous knowledge and understanding of individual plants and their unique characteristics, and it contains important information about plant attributes. The traditional knowledge constituted by these individual plants, including diverse germplasm and traditional medicine resources that have been used for centuries by the ethnic group, is a treasure trove of material and cultural wealth [63]. Therefore, as an important part of ethnic and cultural diversity, folk botanical nomenclature is extremely relevant in biodiversity conservation practices [64]. Second, from the overall perspective of biodiversity conservation, ethnobiological nomenclature reflects the relationship between living organisms and habitats. It is the indigenous epistemology of a complex natural system involving individual organisms and the environment. The use and knowledge of the folk nomenclature of living organisms permit people with non-scientific backgrounds to participate in biodiversity conservation efforts [65]. Many studies have investigated the relationship between cultural diversity and biodiversity, and the positive effect of regional traditional cultures on biodiversity conservation has been widely recognised in the scientific community [66]. For example, studies have shown that biodiversity and cultural diversity overlap in their geographical distribution [67, 68]. For the Yi people in Xiaoliangshan, naming plant species is a rich cultural tradition that was formed as a means of managing and using local plant resources. This traditional knowledge is essential for the protection and sustainable development of local biodiversity.

The folk botanical nomenclature of the Yi people in Xiaoliangshan is an integral part of their traditional knowledge, and it needs to be preserved for future generations. In recent years, accelerated urbanisation and the introduction of foreign culture have greatly affected the traditional knowledge of the Yi people in 
Xiaoliangshan. One manifestation of this trend is the increasing economic migration of young people to large cities [69] and their gradual assimilation into urban society; they thus have fewer opportunities to use their native $\mathrm{Yi}$ language. Due to the assimilation process between the Yi people and the Chinese culture, the language is being increasingly affected. In addition, young people from the Yi ethnic group remaining in Xiaoliangshan now use many Chinese loanwords due to the internet and other mass media usage. Certain popular internet terms have already become an integral part of their language on a large scale, and these are gradually replacing the Yi language [70]. Furthermore, under the recent Poverty Alleviation Resettlement policy, many Yi ethnic group members have been relocated from the mountains to urban areas [71]. The most significant consequence of these above factors is the loss of the local language, and language is the core of culture and the means of transmitting traditional knowledge.

The indigenous nomenclature of plant species is a proper naming system that reflects the rules of the local Yi language. Some studies have shown that the loss of native languages in indigenous communities impairs the transfer of traditional knowledge between different generations, lowers their sense of ethnic identity, and adversely affects the mental and physical health of the indigenous people [72]. In Xiaoliangshan, the loss of the traditional Yi knowledge is obvious; for example, during the interviews conducted in this study, we found that the names of many wild plants commonly collected during the Great Famine in China in the 1960s [73] are now only known by a few aged community members. In addition, the names of plants that are still commonly used for medicinal purposes or as feed are only known by middle-aged and older community members. When shown photographs of different plants, the younger community members recognised the plants, but either could not name them in the Yi language or they only knew the names used by the Han ethnic group, even though their parents were very familiar with and used these plants.

This gradual loss of ethnobotanical names equates to a loss of traditional knowledge and ethnic culture. Studies have shown that the potential for humans to acquire resources from nature through language will become increasingly difficult with the loss of particular languages. Because indigenous languages are closely related to the pharmaceutical knowledge of ethnic groups, it is believed that the demise of indigenous languages will have a greater impact on pharmaceutical knowledge than on the loss of biodiversity [74]. The use of folk botanical names enables us to harness benefits from natural plant resources. Therefore, from the perspective of cultural heritage, creating standardised records of the ethnobotanical nomenclature of the $\mathrm{Yi}$ people in Xiaoliangshan and the rules they used to name plants is critical for preserving this valuable traditional knowledge.

\section{Conclusions}

The Yi ethnic group lives in Xiaoliangshan within north-western Yunnan and in the Daliangshan. Due to the challenging geography and the lack of transportation to the region, the Yi people in Xiaoliangshan historically had very little contact with the outside world. This study used ethnological research methods to document the indigenous plant nomenclature of 226 locally used plant species belonging to 178 genera and 107 families. The nomenclature used by the Yi people in Xiaoliangshan has either a binomial or non-binomial name structure, and four primary factors are used to name plant species: plant characteristics, plant habitat, plant-use, and cultural attributes. The folk names of plants and their corresponding scientific names have the 
following three types of relationships: one plant name for one plant species, two plant names for one plant species, and one plant name for multiple plant species. The folk nomenclature of the Yi ethnic group in Xiaoliangshan has both similarities and differences to that of the Yi group in the Daliangshan, and this is attributed to the relationship between the vitality of the ethnic culture, the extent to which traditional knowledge is practiced by the ethnic community, and the geographical environment in which they live. This study of the folk botanical nomenclature of the Yi ethnic group in Xiaoliangshan will help promote the preservation of traditional knowledge and biodiversity conservation in this area. However, this study only focused on an analysis of ethnobotanical nomenclature, and further research is thus needed to determine whether similar nomenclature rules are used for other living organisms, such as animals and fungi.

\section{Declarations}

\section{Acknowledgements}

We are thankful to the Yi People of the field survey for their kind cooperation and time. The scholars who have made great contributions to the research field of Traditional Knowledge of Yi People are also acknowledged with much gratitude. We would like to say " $\triangle \mathbb{Q}_{\mathbb{Z}}$ "(thank you) to those who helped us.

\section{Authors' contributions}

Yu-Hua Wang organized the study team and provided technical support and guidance. Yu-Hua Wang, Yu Zhang, and Yi-Won A.D designed and executed the research plan. Yi-Won A.D recorded and organized the data and wrote the manuscript. Yi-Won A.D, Xiao-Yong Ding and Chang-An Guo identified the specimen and checked the information. All authors took part in the field works. All authors were involved in the drafting and revision of the manuscript and approved the final revision.

\section{Funding}

The study was funded by "the Second Tibetan Plateau Scientific Expedition and Research (No. 2019QZKK0502)".

\section{Ethics approval and consent to participate}

The authors asked for permission from the local authorities and the people interviewed to carry out the study.

\section{Consent for publication}

The people interviewed were informed about the study's objectives and the eventual publication of the information gathered, and they were assured that the informants' identities would remain undisclosed. 


\section{Competing interests}

The authors declare that they have no competing interests.

\section{Availability of data and materials}

Please contact author for data requests.

\section{References}

1. Berlin. Folk systematics in relation to biological classification and nomenclature. Annu Rev Ecol Syst. 1973; 4 (1): 259-271. http://www.jstor.org/stable/2096813.

2. Xu ZF, Yan HD, Duan QW, HF Z. Dai plant vernacular names and explanations in Xishuangbanna, Yunnan. Beijing: Science Press; 2015.

3. Wang J, Seyler BC, Ticktin T, Zeng Y, Ezhu Z. Indigenous botanical nomenclature used by the Yi people in Liangshan Prefecture, Sichuan Province, China. Econ Bot. 2019; 73 (3): 325340. https://doi.org/10.1007/s12231-019-09461-4.

4. Berlin B, Breedlove DE, Raven PH. Folk taxonomies and biological classification. Science. 1966; 154 (3746): 273-275. https://doi.org/10.1126/science.154.3746.273.

5. Berlin B, Breedlove DE, Raven PH. General principles of classification and nomenclature in folk biology. Am. Anthropol. 1973; 75 (1): 214-242. http://www.jstor.org/stable/672350.

6. Breedlove DE, Laughlin RM. The flowering of man: a Tzotzil botany of Zinacantán. Washington: Smithsonian Institution Press; 1993.

7. Pei SJ, Huai HY. Ethnobotany. Shanghai: Shanghai publisher of Science and Technology; 2007.

8. Wang J, Liu H, Lei HG. Participatory Approach for rapid assessment of plant diversity through a folk classification system in a tropical rainforest: Case study in Xishuangbanna, China. Conserv Biol. 2004; 18 (4):1139-1142. https://doi.org/10.1111/j.1523-1739.2004.00075.x.

9. Xu ZF HY. Research on plant folk nomenclature and taxonomic system of Xishuangbanna Dai nationality. Acta Bot. Yunnanica. 1991 (04): 383-388+390-389.https://doi.org/10.1007/BF02919155.

10. ZF X. Dai People's botanical knowledge: Nomenclature-meaning and classification. Plant Diversity Resour. 2015; 37 (01): 39-45. https://doi.org/10.7677/ynzwyj201514056.

11. Khasbagan, Soyolt. Indigenous knowledge for plant species diversity: a case study of wild plants' folk names used by the Mongolians in Ejina desert area, Inner Mongolia, P. R. China. J Ethnobiol. Ethnomed. 2008; 4 (1): 2-2. https://doi.org/10.1186/1746-4269-4-2.

12. Soyolt, Galsannorbu, Yongping, Wunenbayar, Liu GH, Khasbagan. Wild plant folk nomenclature of the Mongol herdsmen in the Arhorchin national nature reserve, Inner Mongolia, PR China. J Ethnobiol. Ethnomed. 2013; 9 (1): 30-30. https://doi.org/10.1186/1746-4269-9-30.

13. Mandakh U, Battseren M, Ganbat D, Ayanga T, Adiya Z, Borjigidai A, et al. Folk nomenclature of plants in Cistanche deserticola-associated community in South Gobi, Mongolia. Plant Diversity. 2020; 42 (6): $434-$ 
442. https://doi.org/10.1016/j.pld.2020.09.008.

14. Ma CW, Mao XL. Cultural Implication of Plants: A study of the bimo ritual of the Yi as practiced in Xiaoliangshan. Thinking. 2021; 47 (01): 80-92. https://doi.org/10.3969/j.issn.1001-778X.2021.01.010.

15. Stokstad E. Mountains and monsoons created Tibetan biodiversity. Science. 2020; 369 (6503): 493493.https://doi.org/10.1126/science.369.6503.493.

16. Li XW, J. L. A preliminary floristic study on the seed plants from the region of Hengduan mountain. Acta Bot Yunnanica. 1993 (03): 217-231. http://ir.xtbg.org.cn/handle/353005/1518.

17. Ju Y, Zhuo JX, Liu B, Long CL. Eating from the wild: diversity of wild edible plants used by Tibetans in Shangri-la region, Yunnan, China. J Ethnobiol Ethnomed. 2013; 9 (1): 28. https://doi.org/10.1186/17464269-9-28.

18. Wang J, Seyler BC, Ticktin T, Zeng YG, Ayu KD. An ethnobotanical survey of wild edible plants used by the Yi people of Liangshan Prefecture, Sichuan Province, China. J Ethnobiol Ethnomed. 2020; 16 (1): 10.https://doi.org/10.1186/s13002-019-0349-5.

19. Yang LX, Ahmed S, Stepp JR, Mi K, Zhao YQ, Ma JZ, et al. Comparative homegarden medical ethnobotany of Naxi healers and farmers in Northwestern Yunnan, China. J Ethnobiol Ethnomed. 2014; 10 (1): 6-6. https://doi.org/10.1186/1746-4269-10-6.

20. Jiari MJ. Ethnic relations and regional politics in the legend of Yi nationality migration in Xiaoliangshan, Yunnan province in the late Qing Dynasty: an attempt to get rid of fei Xiaotong's Confusion. J Southwest Minzu University (Humanities and Social Science). 2018; 39 (02): 62-72. https://doi.org/10.3969/j.issn.1004-3926.2018.02.009.

21. Zheng CJ. The Yi nationality family branch system and its change in Xiaoliangshan, Yunnan province. $J$ Southwest Minzu University (Humanities and Social Science). 2000 (S3): 125-130.

22. Jiarimuji. A study on the formation village space in the Xiaoliangshan Yi area of Yunnan: A dialogue with prasenjit Duara's "culture nexus of power". Ethno- National Studies. 2012 (01): 40-50+109.

23. Li Z, Yang ZX. Proposes to forest resources investigation and forestry development in Ninglang County of Yunnan Province. Forest Engineering. 2014; 30 (06): 22-26.https://doi.org/10.3969/j.issn.1001005X.2014.06.006.

24. Ding Y, He YZ. Study on soil classification and improvement in Ninglang County. Jiangxi Agriculture. 2019 (04): 52-53. https://doi.org/10.3969/j.issn.1674-1479.2019.04.044.

25. Xu SY. Anthropological Analysis of changes of livelihood pattern of Yi ethnic minority in Xiaoliangshan Area: A Case Study of Paomaping Town of Lijiang City. J Honghe University. 2021; 19 (01): 40-43. https://doi.org/10.13963/j.cnki.hhuxb.2021.01.010.

26. Wang Y, Li HJ. A study on Yi people's wooden houses in Xiaoliangshan of Yunnan. J Wenshan University. 2021;34 (01): 50-55. https://doi.org/10.3969/j.issn.1674-9200.2021.01.010.

27. Jia YZ. The diet culture of Yi nationality. Chengdu: Sichuan University Press; 1994.

28. Yang ZX, Bibu RF. Interpret the relationship between "snow nation" and human beings in the concept of Yi nationality. J Southwest Minzu University (Humanities and Social Science). 2011; 32 (12): 64-67. https://doi.org/10.3969/j.issn.1004-3926.2011.12.014.

29. Zhang ZH. Bimo and Suni of the Yi religion in southwest China. Religious Studies. 2012 (04): $223-231$. 
30. Zhang ZH. Ancestral worship and multi-belief system of Yi religion in Southwest China. Religious Studies. 2011 (04): 186-192

31. Wang CY. A comparative study of Yi dialects. Chengdu: Sichuan Nationalities Publishing House; 2003.

32. Editoral Committee of Flora of China. Flora of China. Beijing: Science Press; 2013.

33. Wang JX, Liu HM, Hu HB, Gao I. Participatory Approach for rapid assessment of plant diversity through a folk classification system in a tropical Rainforest: Case study in Xishuangbanna, China. Conserv Biol. 2004; 18 (4): 1139-1142. https://doi.org/10.1111/j.1523-1739.2004.00075.x.

34. Chen S. Plant naming and Mongolian culture. J Inner Mongolia Normal University. 1992 (3): 5263.https://doi.org/CNKI:SUN:NMSB.0.1992-03-007.

35. Kolosova V, Svanberg I, Kalle R, Strecker L, Özkan AMG, Pieroni A, et al. The bear in Eurasian plant names: motivations and models. J Ethnobiol Ethnomed. 2017; 13 (1):

14.https://doi.org/10.1186/s13002-016-0132-9.

36. Soyolt, Galsannorbu, Yongping, Wunenbayar, Liu G, Khasbagan. Wild plant folk nomenclature of the Mongol herdsmen in the Arhorchin national nature reserve, Inner Mongolia, PR China. BioMed Central. 2013; 9 (1): 30-30. https://doi.org/10.1186/1746-4269-9-30.

37. Li RT. The study of plant nouns based on animals. J Henan Normal University. 2008; 35 (5): 3.https://doi.org/10.16366/j.cnki.1000-2359.2008.05.026.

38. Gao W. Acculturation and Livelihood Choice: an ecological anthropological study of animal husbandry economy in Yi Nationality region. Heilongjiang Animal Science and Veterinary Medicine. 2014; (15): 264266.

39. Cao WJSGL, Te BQ. Exploration on the mutual infiltration teaching of Mongolian medicinal botany and Mongolian folk traditional medicine knowledge - a case study of medicinal plant folk name. J Medicine \& Pharmacy of Chinese Minorities, 2015; 21 (10): 48-51. https://doi.org/10.16041/j.cnki.cn151175.2015.10.025.

40. Shi L, Huang Y. Cultural connotation in classifying and naming Dong plants. J Baise University. $2017 ; 30$ (02): 42-51.

41. Xian XL. On the Motivation of plant-naming in Sichuan Chorography. J Neijiang Normal University 2008; (11): 102-106. https://doi.org/10.3969/j.issn.1671-1785.2010.11.025.

42. Tan HJ, Chen YY. On the characteristics of plant nomenclature in Guangzhou dialect. J Baicheng Normal University. 2020; 34 (04): 51-55.

43. Tan H, J., Lei HY. Language Cognitive Analysis of plant names in modern chinese. J Changchun University. 2020; 30 (09): 41-46. https://doi.org/10.3969/j.issn.1009-3907.2020.05.009.

44. Zhang KN. From the barefoot doctor to the country doctor. Kunming: Yunnan People's Publishing House; 2002.

45. Xu S, Hu D. Barefoot Doctors and the "Health Care Revolution" in rural China: A study centered on Shandong province. Endeavour. 2017; S0160932717300662.https://doi.org/10.1016/j.endeavour.2017.06.004.

46. Chen $\mathrm{H}$. The Conceptual Metaphors of "A PLANT IS A HUMAN BEING": A contrastive study based on Chinese and English plant names. Foreign Language and Literature. 2014; 30 (05): 81- 
87. https://doi.org/10.3969/j.issn.1674-6414.2014.05.014.

47. Du J, WU J, Yang GL, Li YQ. Practice of Chuxiong Normal University in cultivating musicianship for “Application\orientated” primary School Teachers. J Chuxiong Normal University. 2016; 031 (011): 84$91,101$.

48. Yang TS. Plant and culture: Another interpretation of human history. J Jishou University (Social Sciences). 2012; 33 (01): 1-7. https://doi.org/10.3969/j.issn.1007-4074.2012.01.001.

49. Wu ZY. Flora of Yunnan. Vol.6, seed plants. Beijing: science press 1995.

50. Yan QJ. Research on Yi people's food culture from the ecological perspective. J Qingdao Agricultural University (Social Science). 2020; 32 (04): 87-90. https://doi.org/10.3969/J.ISSN.16741471.2020.04.016.

51. Editoral Committee of Flora of China. Flora of China. Beijing: science press 1990.

52. Lan YH. The ancient Chinese method of naming plants and animals as found in the SHI MING OF THE BEN CAO GANG MU. Studies in the History of Natural Sciences. 1989; 8 (02): 166-170.

53. Group BYLT. The history of Yi nationality in southwest of China. Guiyang: Guizhou Nationalities Publishing House; 1988.

54. Shang YH. The belief culture of the minority of Yi nationality in the Micro Perspective--A study on the relic worship of plants of Micha people of Yi nationality. J Guizhou University of Engineering Science. 2019; 37 (06): 54-59. https://doi.org/10.3969/j.issn.1673-7059.2019.06.010.

55. Song YJ, Long CL. Traditional knowledge and culture of buckwheat. Science. 2019; 71 (02): 912+14.https://doi.org/10.3969/j.issn.0368-6396.2019.02.004.

56. Zhu SZ. Plantation and distribution of the main crops in the Liangshan Yis ethnic areas in Sichuan Province in history. Agricultural History of China. 2006. https://doi.org/10.3969/j.issn.10004459.2006.02.002.

57. Editoral Committee of Flora of China. Flora of China. Beijing: Science Press; 1974.

58. Editoral Committee of Flora of China. Flora of China. Beijing: Science press; 1990.

59. Quave CL, Pieroni A. A reservoir of ethnobotanical knowledge informs resilient food security and health strategies in the Balkans. Nature Plants. 2015; 1 (2): 14021. https://doi.org/10.1038/nplants.2014.21.

60. Myers N, Mittermeier RA, Mittermeier CG, da Fonseca GAB, Kent J. Biodiversity hotspots for conservation priorities. Nature. 2000; 403 (6772): 853- 858.https://doi.org/10.1038/35002501.

61. Zhang Y, Lin GS. An introduction and analysis of biodiversity conservation in Indo-Burma biodiversity hotspot. Landscape Architecture. 2015 (06): 16-24. https://doi.org/10.14085/j.fjyl.2015.06.0016.09.

62. Wang JP, He J. Ethnic traditional culture and biodiversity conservation in Yunnan Province. Journal of West China Forestry Science. $2021 ; 50$ (05): 124-128.

63. Huai HY, Pei SJ. Advances of medical ethnobotany. Chinese Bulletin of Botany. 2002 (02): 129136.https://doi.org/10.16473/j.cnki.xblykx1972.2021.05.018.

64. Xue DY, Guo L. On concepts and protection of traditional knowledge Biodiversity Science. $2009 ; 17$ (02): 135-142. https://doi.org/10.3724/SP.J.1003.2009.08256. 
65. Phaka FM, Netherlands EC, Kruger DJD, Du Preez LH. Folk taxonomy and indigenous names for frogs in Zululand, South Africa. J. Ethnobiol. Ethnomed. 2019; 15 (1): 17. https://doi.org/10.1186/s13002-0190294-3.

66. Shao H, Yang JB, Xue DY. Applications of Wa traditional culture in biodiversity conservation. Biodiversity Science. 2021; 29 (08): 1120-1127. https://doi.org/10.17520/biods.2020480.

67. Gorenflo LJ, Romaine S, Mittermeier RA, Walker-Painemilla K. Co-occurrence of linguistic and biological diversity in biodiversity hotspots and high biodiversity wilderness areas. PNAS. 2012; 109 (21): $8032-$ 8037. https://doi.org/10.1073/pnas.1117511109.

68. Loh J, Harmon D. A global index of biocultural diversity. Ecological Indicators. 2005;5 (3): 231 241.https://doi.org/10.1016/j.ecolind.2005.02.005.

69. LI JP. Investigation of ethnic Minority migrant workers in cities -A case study of Yi nationality migrant workers in Xiegang Town, Dongguan. J Aba Teachers University. 2010; 27 (03): 3639. https://doi.org/10.3969/j.issn.1008-4142.2010.03.010.

70. Li L. Language ecology of Yi nationality in Xiaoliangshan, Yunnan. Culture Journal. 2016 (12): $175-177$.

71. Zhang $X Y$, Wang ZN. An analysis of poverty relief relocation in Ninglang County in post-poverty era. Shanxi Agricultural Economy. 2021 (15): 23-25+28. https://doi.org/10.16675/j.cnki.cn141065/f.2021.15.007.

72. Khawaja., Masud. Consequences and remedies of Indigenous Language loss in Canada. Societies. 2021; 11 (3): 89.https://doi.org/10.3390/soc11030089.

73. Jia YM, Zhu J. A review of the great famine studies by domestic scholars. J Jiangsu University (Social Science Edition). 2015; 17 (02):14-24. https://doi.org/10.13317/j.cnki.jdskxb.2015.015.

74. Cámara-Leret R, Bascompte J. Language extinction triggers the loss of unique medicinal knowledge. PNAS. 2021; 118 (24): e2103683118. https://doi.org/10.1073/pnas.2103683118.

\section{Figures}




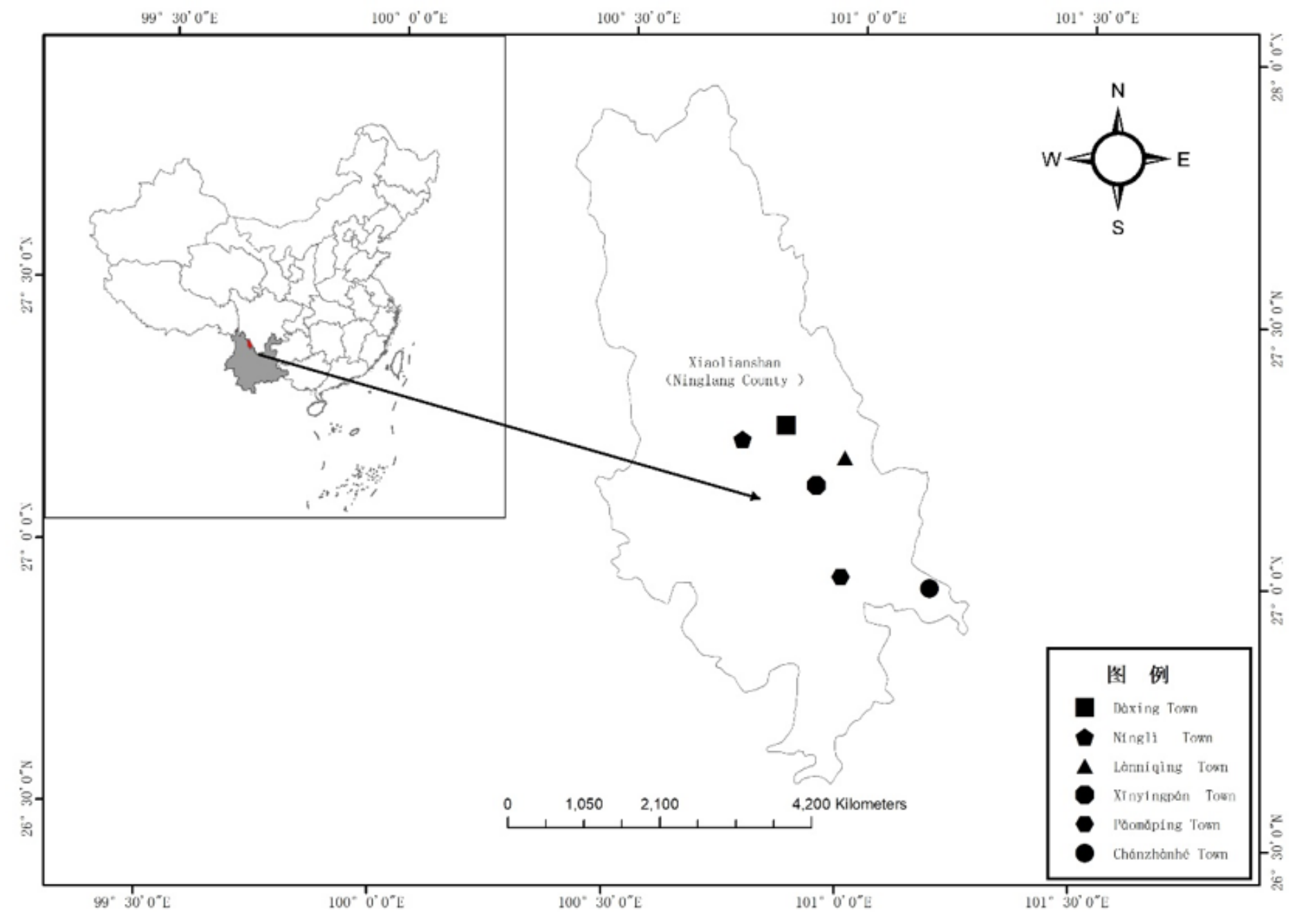

Figure 1

Study area location: Xiaoliangshan, Yunnan Province, China. 
$\square$ Usage $\square$ Plant habitat $\square$ Cultural characteristics $\quad$ Plant characteristics

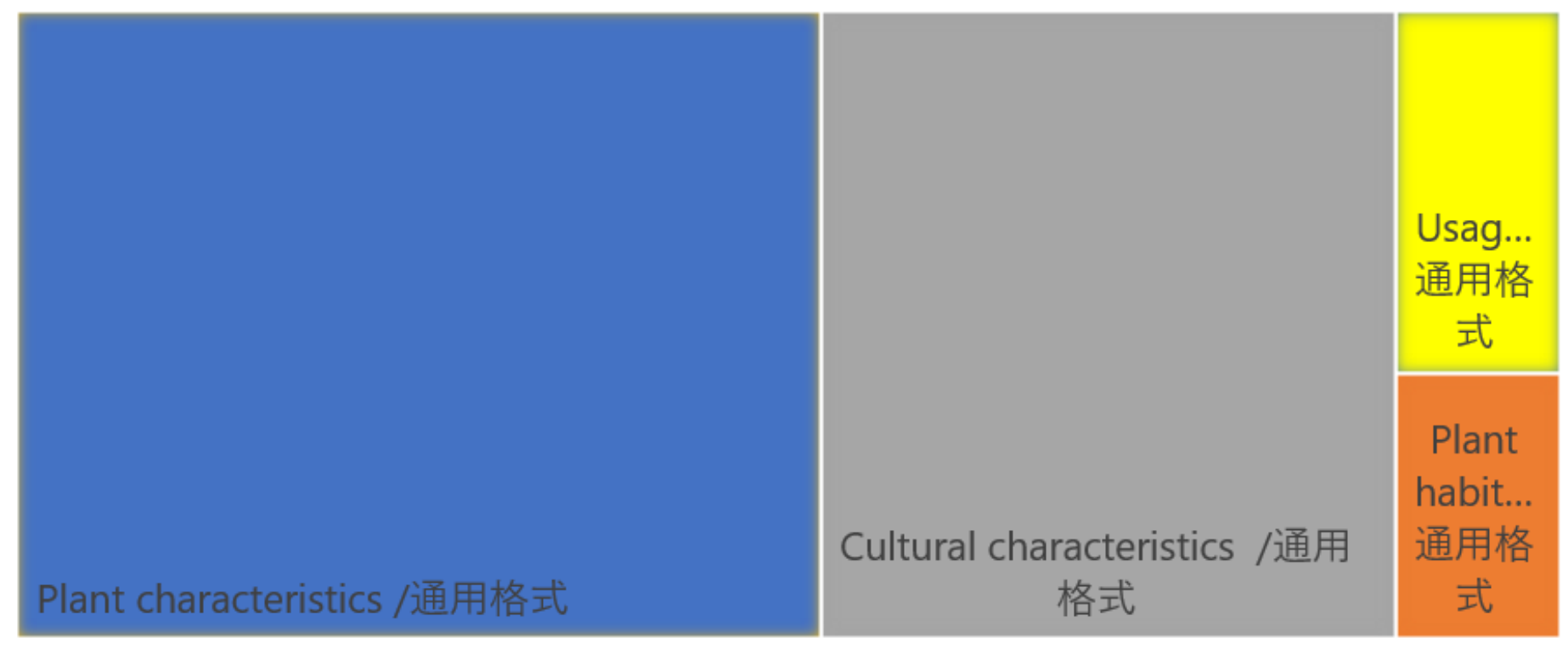

\section{Figure 2}

Folk nomenclature criteria for naming plant species in the Yi community of Xiaoliangshan. Note: The numbers represent the plant species named using each method. 
160

140

120

100

80

67

60

40

20

0

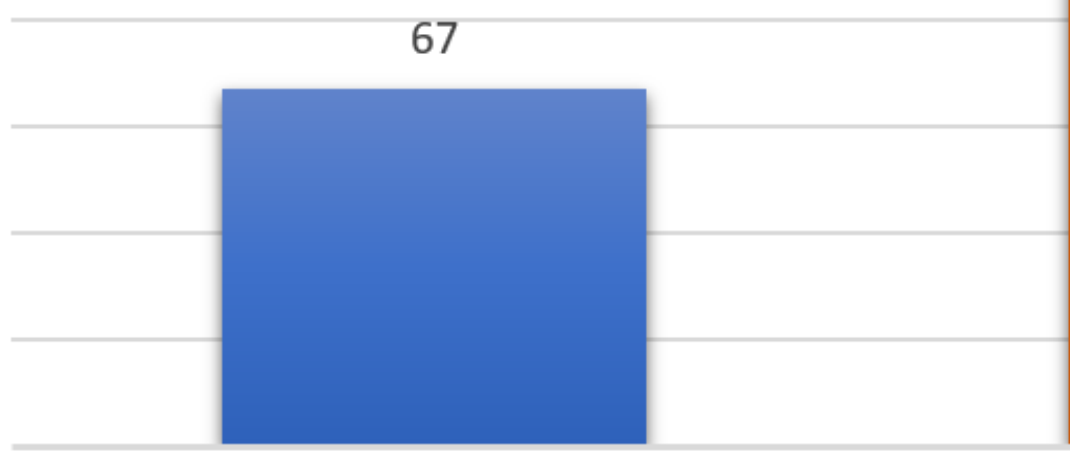

Binomial nomenclature

Non-binomial nomenclature

\section{Figure 3}

The basic structure of folk plant names used by the Yi people in Xiaoliangshan. 


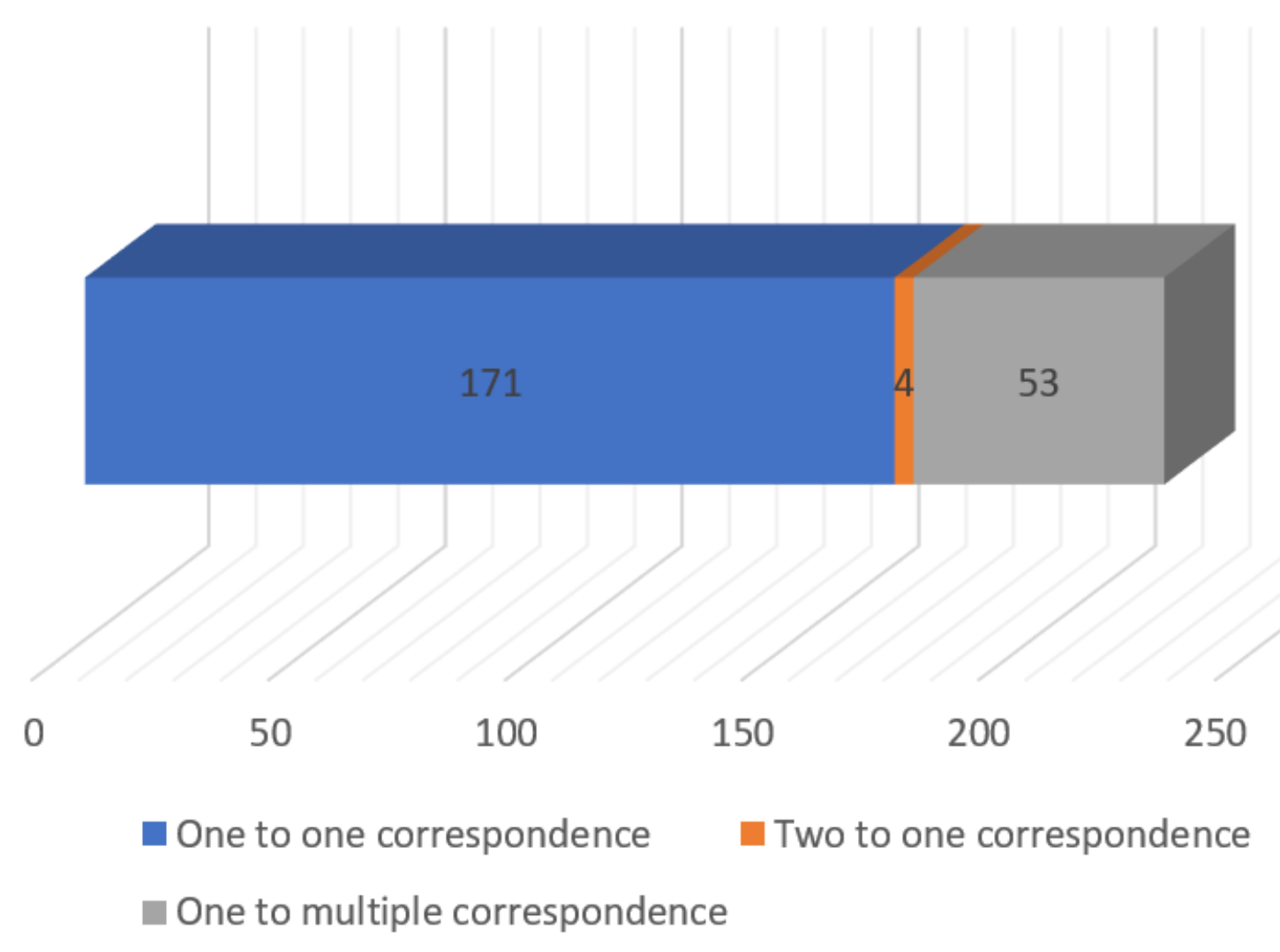

\section{Figure 4}

Correspondence between folk botanical names used by the Xiaoliangshan Yi people and plant species. Note: The different colours represent the corresponding relationships between the folk names of plants used by the Yi people and plant species: blue indicates a one-name-to-one- species relationship; Orange indicates a twonames-to-one-species relationship and grey indicates a one-name-to-multiple-species relationship. 


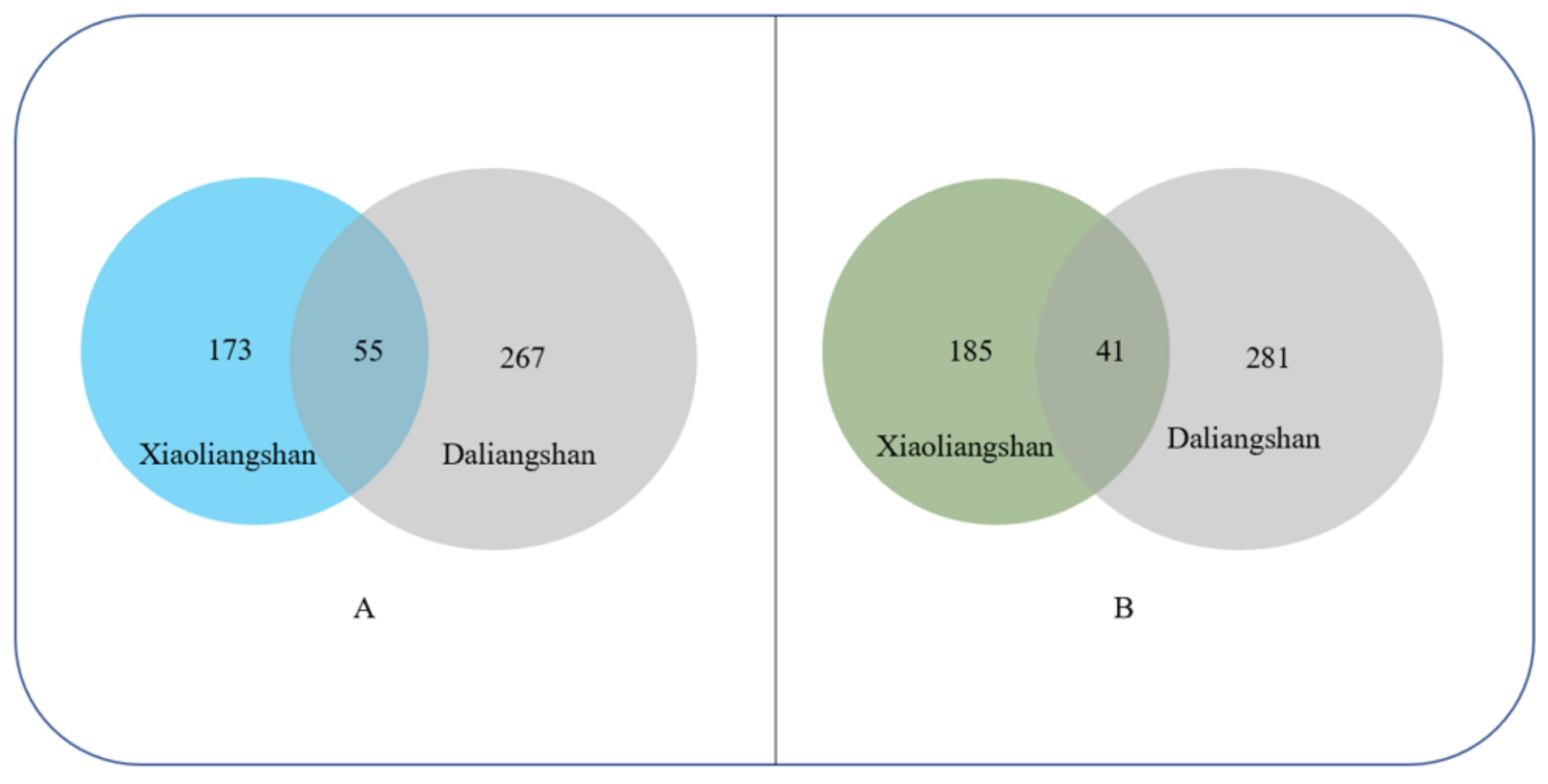

Figure 5

Comparison between the folk botanical nomenclature of the Yi people in Xiaoliangshan and the Yi people in the Daliangshan. Note: A shows the overlap between the names of Yi plants in Xiaoliangshan and those in Daliangshan; B shows the plants used in both places and the overlap. 